\title{
Mechanism design and parameter optimization of a new asymmetric translational parallel manipulator
}

\author{
Yi Yang, Yaqi Tang, Haijun Chen, Yan Peng, and Huayan Pu \\ School of Mechatronic Engineering and Automation, Shanghai University, Shanghai, 200444, China \\ Correspondence: Yan Peng (pengyan@shu.edu.cn) \\ Received: 6 November 2018 - Revised: 18 February 2019 - Accepted: 23 May 2019 - Published: 18 June 2019
}

\begin{abstract}
With the requirement of heavy load for pick-and-place operation, a new 3-DoF asymmetric translational parallel manipulator is invented in this paper. This manipulator is assembled by a kinematic limb with the parallel linear motion elements(PLMEs), and a single loop 2-UPR. Owning to the linear actuators directly connecting the moving and the fixed platforms, this parallel manipulator has high force transmission efficiency, and adapts to pick-and-place operation under heavy load. In this paper, the mobility and singularity are firstly analyzed by screw theory. And the simplified kinematic and dynamic model is established and solved. Secondly, the reaction forces of the prismatic joints in the PLMEs limb are investigated for the mechanism design. Also, the overall performance of the whole manipulator, such as the workspace, condition numbers of Jacobian matrices and motion transmission, etc, are discussed. Thirdly, a compound evaluation function, which involves the factors of workspace volume, transmission efficiency and reaction force, is proposed. In order to obtain a set of better design parameters, the optimization of the 3-DoF translational manipulator is conducted, for the object of maximum of the evaluation function. At last, the prototype is manufactured and experimented to validate the mobility and motion feasibility of this mechanism design.
\end{abstract}

\section{Introduction}

As the need of the industry for 3-DoF translational parallel mechanisms(TPM) in the late 1990s, many these kinds of parallel mechanisms have been researched and developed. In academic, several approaches for the type synthesis of TPMs were investigated, such as methods based on screw theory (Mohamed et al., 1985; Lee et al., 1999; Zhao et al., 2002; Bonev et al., 2003; Huang and Li, 2003; Kong and Gosselin, 2004a; Dai, 2006, 2014; Dai et al., 2006; Wu et al., 2010; Zhao et al., 2017), displacement group theory (Hervé, 1999; Lee et al., 2009), position and orientation characteristic (POC) sets (Yang et al., 2009), generalized function (GF) sets (Gao et al., 2011) and etc. By these means, a number of novel TPMs were invented by Tsai and Joshi (2000); Chablat and Wenger (2003); Liu et al. (2003); Kong and Gosselin (2004b); Jin and Yang (2004); Gogu (2008); Yang et al. (2019) and et al. And the kinematics, dynamics, singularities, stiffness, workspaces for the 3-DOF TPMs were contributed by Carricato and Parenti-Castelli (2002); Li and Xu (2008);
Liu et al. (2017); Kong and Gosselin (2002); Li et al. (2015); Zhang et al. (2017), amongst others.

The actuators among the above TPMs can be divided into 2 primitive types, i.e, rotational actuators and linear actuators. The well-known 3-DoF TPM, Delta robot, is driven by 3 rotational actuators located on the base (Pierrot et al., 1990). Due to its capacity of high speed and high accelerations, this robot has popular usage in picking and packaging in factories. In 1996, Tsai proposed a typical 3-UPU parallel robot, called Tsai manipulator (Tsai and Joshi, 2000). The prismatic joint in each leg is driven by one linear actuator. Compared with the rotational actuators, the linear actuators generally deliver large force at high efficiency due to the simple transmission structure, and are used in a wide range of application in industry, especially in heavy duty equipments. Therefore, in the design of the heavy-load translational parallel manipulator, we choose the linear actuators to drive the moving platform of TPM in this paper.

As aforementioned TPMs, Tsai manipulator can be driven by 3 linear actuators in each leg. Nevertheless, Han et al. 


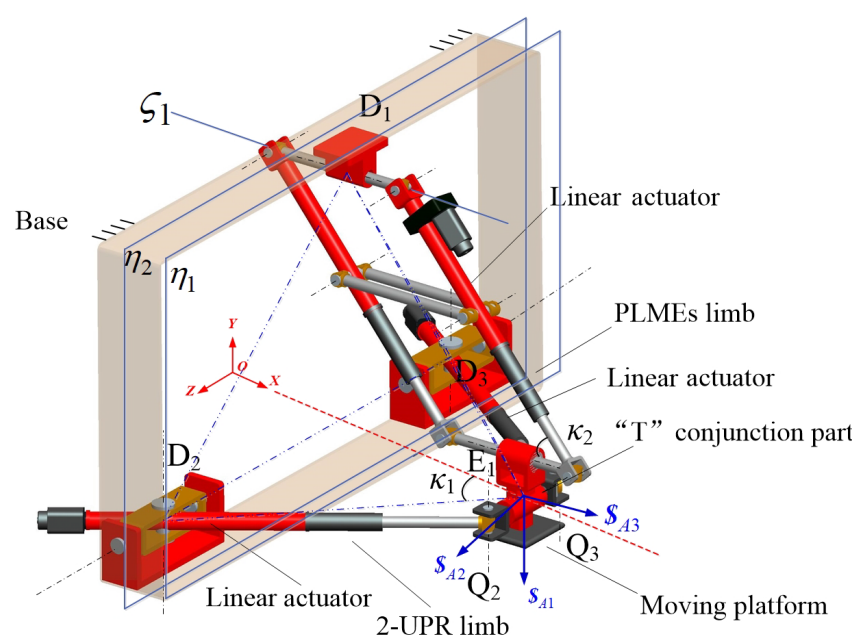

(a)

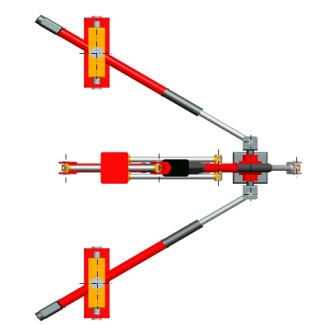

(b)

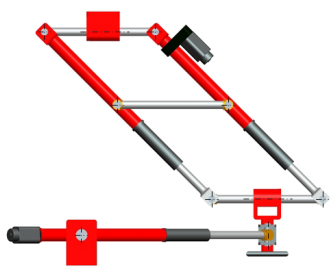

(c)

Figure 1. A 3-DoF translational manipulator with PLMEs limb and single loop 2-UPR: (a) oblique view, (b) top view, (c) side view.

(2002) showed that the small torsions in the legs of 3-UPU generated large deviations in the position of the moving platform. Thus, the applications of the Tsai's manipulator are limited in industry. The "Linear Deltas" has been developed from the standard Delta robot (Bouri and Clavel, 2010). The linear actuators move the parallelograms in each leg up or down, and then lead to the translating of the end platform. Furthermore, Yang et al. (2018) proposed a novel kind of kinematic chains with parallel linear motion elements(PLMEs), and synthesized a class of symmetrical 3T, 3T-1R, and 3R parallel mechanisms by these kinematic limbs. Different from "Linear Delta", the linear actuators in these limbs directly connect the moving platform and the fixed base, which makes the parallel manipulators capable of higher transmission efficiency. Inspired by the above scholars' achievements, we propose a new 3-DoF translational manipulator by combining of PLMEs limb and single loop 2UPR (Peng et al., 2018). This kind of manipulator has advantage of better transmission and simpler structure than other 3-DoF TPMs.

The rest of the paper is organized as follows. In Sect. 2, the structure is elaborated, and the mobility and singularity are analyzed. In Sect. 3, the kinematic and dynamic model is established and solved. In Sect. 4, the reaction forces of the prismatic joints in the PLMEs limb are studied. In Sect. 5, the overall performance of the whole manipulator, such as the workspace, condition numbers of Jacobian matrices and motion transmission, etc, are evaluated. In Sect. 6, a compound evaluation function is proposed. And the parameters of this manipulator are optimized. Finally, in Sect. 7, the prototypes are manufactured and validate the mobility and motion feasibility of this new manipulator.

\section{Structure and mobility of the new manipulator}

The new 3-DoF manipulator is assembled by the parallel linear motion elements(PLMEs) limb and a single loop 2-UPR, as shown in Fig. 1. The moving platform is driven by 3 linear actuators, i.e., one actuator located in PLMEs limb and the other two actuators located in the single loop 2-UPR. To investigate the mobility and singularity of this new manipulator, we firstly analyze the PLMEs limb and the 2-UPR limb individually, by the utilization of screw theory. Secondly, we express the screws of the mechanism by Grassmann line geometry, and obtain the DoF space of the whole manipulator. Furthermore, we also discuss the controllability of the moving platform driven by the 3 selected linear actuators.

As an effective tool, screw theory is widely applied to analyze the mobility, singularity, transmission of mechanisms. A screw is usually represented by the form of Plucker homogeneous coordinates $(L, M, N, P, Q, R)$. In mechanism research, the general term "screw" can be divided into twist $\$$ and wrench $\$^{\mathrm{r}}$. In twist $\$$, the first three components denote an instantaneous angular velocity around an axis. And the last three components denote an instantaneous linear velocity along this axis. In wrench $\$^{\mathrm{r}}$, The first three components denote the resultant force and the last three components denote the resultant moment. If the reciprocal product of the two screws, $\$$ and $\$^{\mathrm{r}}$, equals zero, these two screws are said to be reciprocal. The details can be found in Kong and Gosselin (2004a); Dai et al. (2006); Dai (2014), etc.

\subsection{PLMEs limb}

In Fig. 2, the PLMEs is constructed by two linear motion elements $A_{1} C_{1}$ and $A_{2} C_{2}$. The parallelogram $A_{1} B_{1} A_{2} B_{2}$ guarantees $A_{1} C_{1}$ and $A_{2} C_{2}$ be always parallel. The two inner 


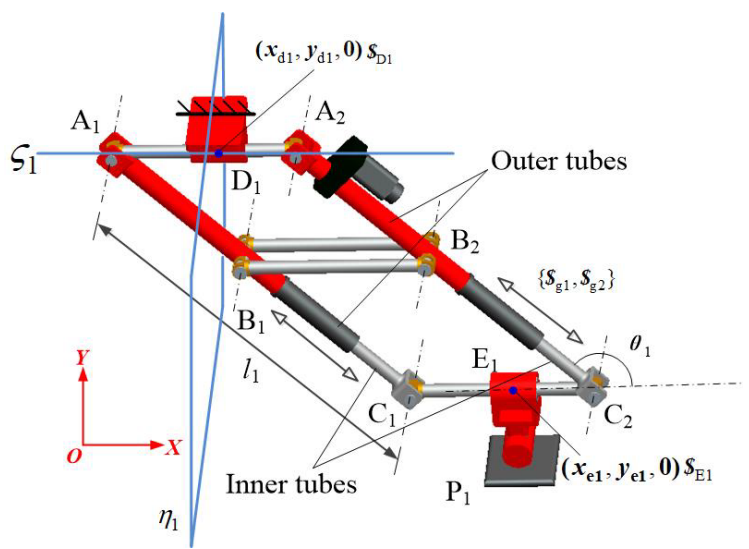

Figure 2. PLMEs limb.

tubes are able to slide along axes $A_{1} B_{1}$ and $A_{2} B_{2}$, respectively.

With reference (Yang et al., 2018), the moving link $C_{1} C_{2}$ generally has 2 translational DoFs without consideration of the revolute joints $D_{1}$ and $E_{1}$. The PLMEs linkage $A_{1} A_{2} C_{1} C_{2}$ can be regarded as a generalized kinematic pair, whose twist is denoted as $\left\{\$_{\mathrm{g} 1}, \$_{\mathrm{g} 2}\right\}$. Adding the revolute joints $D_{1}$ and $E_{1}$, the twist of the platform $P_{1}$ is then written as in Eq. (1) if the PLMEs linkage is not in a singular configuration.

$\$_{\mathrm{p} 1}:=\left\{\begin{array}{l}\$_{\mathrm{D} 1}=\left[\begin{array}{llllll}1 & 0 & 0 & 0 & 0 & -y_{\mathrm{d} 1}\end{array}\right] \\ \$_{\mathrm{g} 1}=\left[\begin{array}{llllll}0 & 0 & 0 & \cos \theta_{1} & \sin \theta_{1} & 0\end{array}\right] \\ \$_{\mathrm{g} 2}=\left[\begin{array}{llllll}0 & 0 & 0 & -l_{1} \sin \theta_{1} & l_{1} \cos \theta_{1} & 0\end{array}\right] \\ \$_{\mathrm{E} 1}=\left[\begin{array}{llllll}1 & 0 & 0 & 0 & 0 & -y_{\mathrm{e} 1}\end{array}\right]\end{array}\right.$

Its corresponding reciprocal screw $\$_{\mathrm{sp}}^{\mathrm{r}}$ can be obtained as

$\$_{\mathrm{p} 1}^{\mathrm{r}}:=\left\{\begin{array}{l}\$_{\mathrm{sp} 1}^{\mathrm{r} 1}=\left[\begin{array}{llllll}0 & 0 & 0 & 0 & 0 & 1\end{array}\right] \\ \$_{\mathrm{sp} 1}^{\mathrm{r} 2}=\left[\begin{array}{llllll}0 & 0 & 0 & 0 & 1 & 0\end{array}\right]\end{array}\right.$

Equation (2) can be represented by the constraint space graph as shown in Fig. 5a. When $\theta_{1}= \pm \pi / 2$, this configuration is a singularity. The $Z$ axis rotational constraint is absent and the constraints reduce to only one. The kinematic limb has an extra instantaneous rotation about $Z$ axis. Considering the rotating of this limb along $X$ axis, this kind of singular configurations are all distributed on the plane $\eta_{1}$, as shown in Fig. 2. When $\theta_{1}=0, \pi$, the axes of the revolute joints $D_{1}$ and $E_{1}$ are collinear and the twists $\$_{\mathrm{D} 1}$ and $\$_{\mathrm{E} 1}$ are correlation. In this case, the moving platform has an additional instantaneous constraint to prevent it from translating along $Z$ axis. This singular configuration is on the line $\varsigma_{1}$, as shown in Fig. 2.

In the above PLMEs limb, the mid-link $B_{1} B_{2}$ connects the 2 outer tubes of the linear motion elements, as shown in Fig. 3a. In actual design, we can change the mid-link $B_{1} B_{2}$ from the outer tubes to the inner tubes, as shown in Fig. $3 \mathrm{~b}$. The parallelogram $B_{1} C_{1} B_{2} C_{2}$ guarantees $A_{1} C_{1}$ and $A_{2} C_{2}$ be always parallel. Thus, the mobility, constraint and singularity are as the same as the one in Fig. 3a. In another case, as shown in Fig. 3c, the mid-link $B_{1} B_{2}$ is fixed on the ground. The two sliders on the points $B_{1}$ and $B_{2}$ are jointed with the mid-link. The two linear guides $A_{1} C_{1}$ and $A_{2} C_{2}$ can slide on $B_{1}$ and $B_{2}$. The linkage $A_{1} C_{1} A_{2} C_{2}$ is a parallelogram. Therefore, the PLMEs presented in Fig. $3 \mathrm{c}$ has also the same kinematic characteristics with the above two ones. From the kinematic point of view, these three PLMEs limbs presented in Fig. 3 are all equivalent.

\subsection{Single Loop 2-UPR}

This single loop is constructed by 2 UPR limbs, as illustrated in Fig. 4. In Limb $D_{2} Q_{2}$, one axis of the universal joint $D_{2}$ is perpendicular with $X-Y$ plane. The axis orientation is $s_{1}=(0,0,1)$. The other axis is perpendicular with the plane formed by the vectors $\boldsymbol{s}_{1}$ and $\left(\boldsymbol{p}_{Q_{2}}-\boldsymbol{p}_{D_{2}}\right)$, where $\boldsymbol{p}_{Q_{2}}$ and $\boldsymbol{p}_{D_{2}}$ are the coordinates of the points $Q_{2}$ and $D_{2}$. This axis orientation can be written as $\boldsymbol{s}_{2}=\boldsymbol{s}_{1} \times\left(\boldsymbol{p}_{Q_{2}}-\boldsymbol{p}_{D_{2}}\right)$. The axis of another joint $Q_{2}$ is parallel with the vector $\boldsymbol{s}_{2}$. The other Limb $D_{3} Q_{3}$ has the similar condition with $D_{2} Q_{2}$.

For Limb $D_{2} Q_{2}$, the twist of each kinematic pair can be written as

$\left\{\begin{array}{l}\$_{11}=\left(\begin{array}{ll}\boldsymbol{s}_{1} ; & \boldsymbol{p}_{\mathrm{D} 2} \times \boldsymbol{s}_{1}\end{array}\right) \\ \$_{12}=\left(\begin{array}{ll}\boldsymbol{s}_{2} ; & \boldsymbol{p}_{\mathrm{D} 2} \times \boldsymbol{s}_{2}\end{array}\right) \\ \$_{13}=\left(\begin{array}{ll}0 ; & \boldsymbol{p}_{Q_{2}}-\boldsymbol{p}_{\mathrm{D} 2}\end{array}\right) \\ \$_{14}=\left(\begin{array}{cc}\boldsymbol{s}_{2} ; & \boldsymbol{p}_{Q_{2}} \times \boldsymbol{s}_{2}\end{array}\right)\end{array}\right.$

Through calculating the nullity of the above screws, the corresponding reciprocal screw of limb $D_{2} Q_{2}$ can be obtained as

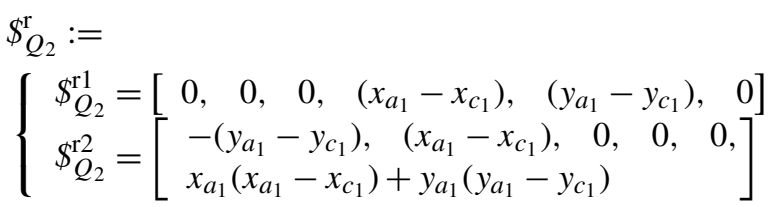

In the same way, the wrench of limb $D_{3} Q_{3}$ is

$$
\begin{aligned}
& \$_{Q_{3}}^{\mathrm{r}}:=
\end{aligned}
$$

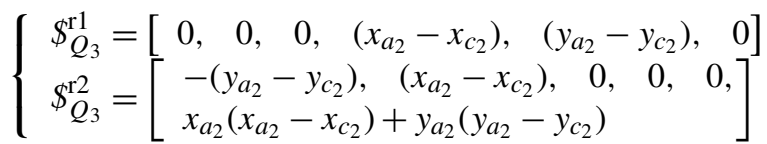

If $Q_{2} Q_{3}$ and $D_{2} D_{3}$ are parallel with each other and both perpendicular with $X-Y$ plane, the coordinates of the joints satisfy

$x_{a_{2}}=x_{a_{1}}, \quad y_{a_{2}}=y_{a_{1}}, \quad x_{c_{2}}=x_{c_{1}}, y_{c_{2}}=y_{c_{1}}$

Substituting the above condition into Eqs. (4) and (5), and using the algebra operation of reciprocal product, the twist of link $Q_{2} Q_{3}$ which is supported by the limbs $D_{2} Q_{2}$ and 


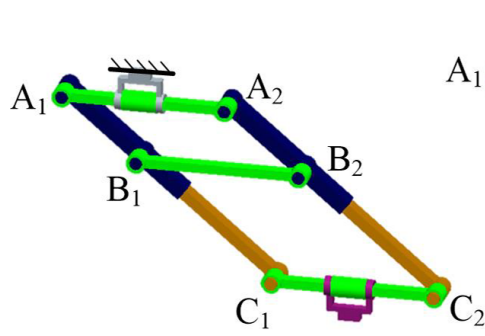

(a)

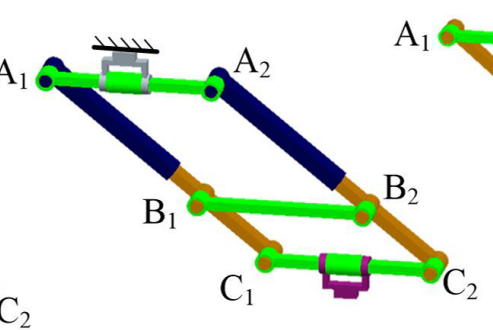

(b)

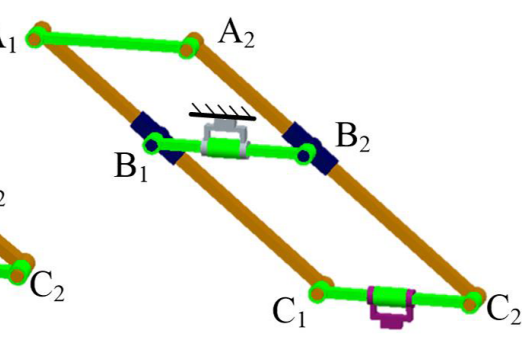

(c)

Figure 3. Three equivalent PLMEs limbs: (a) mid-link connecting outer tubes, (b) mid-link connecting inner tubes, (c) mid-link fixing on the ground.

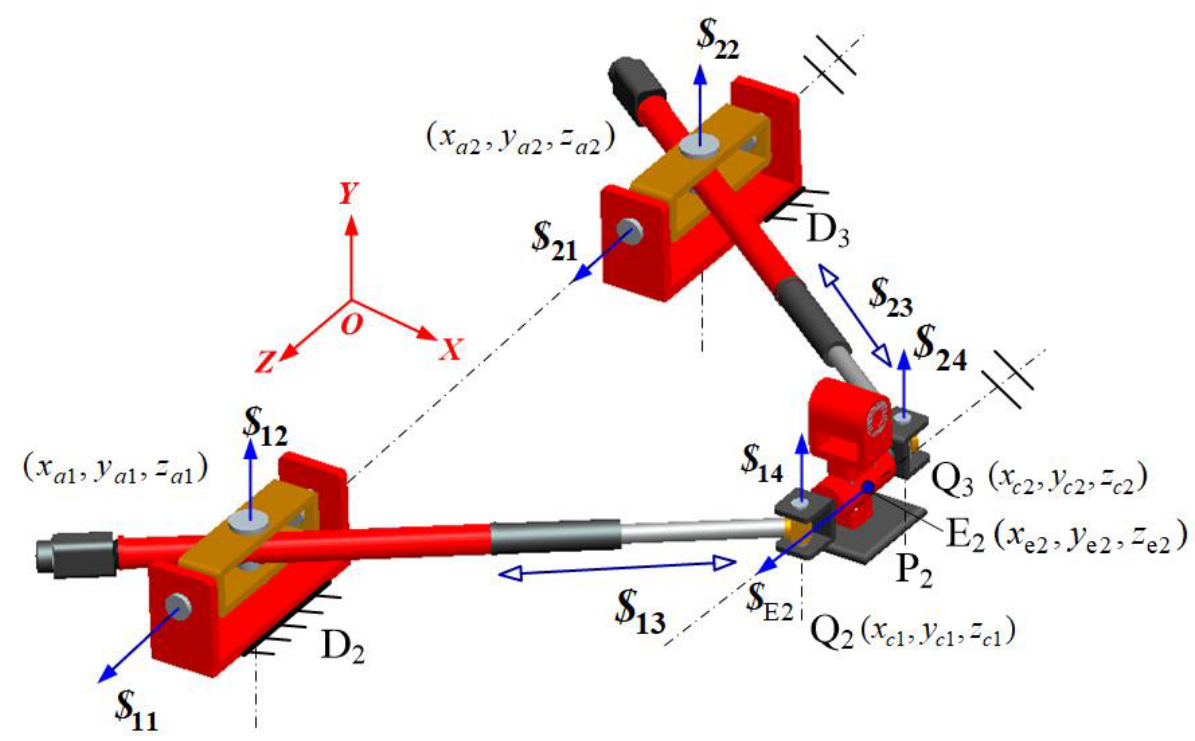

Figure 4. Single Loop 2-UPR.

$D_{3} Q_{3}$ can be figured out. The results are as following

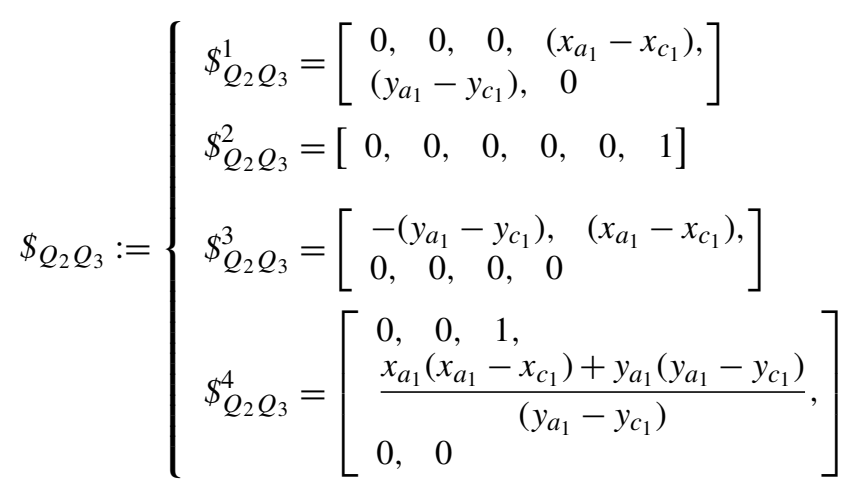

Within the consideration of the revolute pair $E_{2}$ attached on the link $Q_{2} Q_{3}$, the twist of the platform $P_{2}$ can then be expressed as

$\$_{p_{2}}:=\left\{\begin{array}{l}\$_{Q_{2} Q_{3}} \\ \$_{\mathrm{E} 2}=\left[\begin{array}{llllll}0, & 0, & 1, & y_{e_{2}}, & -x_{e_{2}}, & 0\end{array}\right]\end{array}\right.$
By solving the nullity of Eq. (8), the wrench of the moving platform $P_{2}$ can be obtained.

$\$_{p_{2}}^{\mathrm{r}}=\left[\begin{array}{llllll}0 & 0 & 0 & x_{a_{1}}-x_{c_{1}} & y_{a_{1}}-y_{c_{1}} & 0\end{array}\right]$

According to Eq. (9), the corresponding constraint space graph of the moving platform $P_{2}$ is plotted as shown in Fig. 5b. Moreover, we substitute $S$-joint for $U$-joint. The single loop 2-SPR is instead of the loop 2-UPR. By repeating the above analysis process, it can be derived that the wrench of the moving platform $P_{2}$ in this case still equals Eq. (9). This means that the loops 2-UPR and 2-SPR are equivalent and they can be swapped with each other for the requirements.

\subsection{Whole manipulator}

The PLMEs limb and the Loop 2-UPR are connected by a " $T$ " shaped conjunction part, as shown in Fig. 1. The mobility of the whole manipulator can be deduced by Grassmann line geometry and the line-graph method. Grassmann line geometry is a systematic theory in mathematics, which can be 


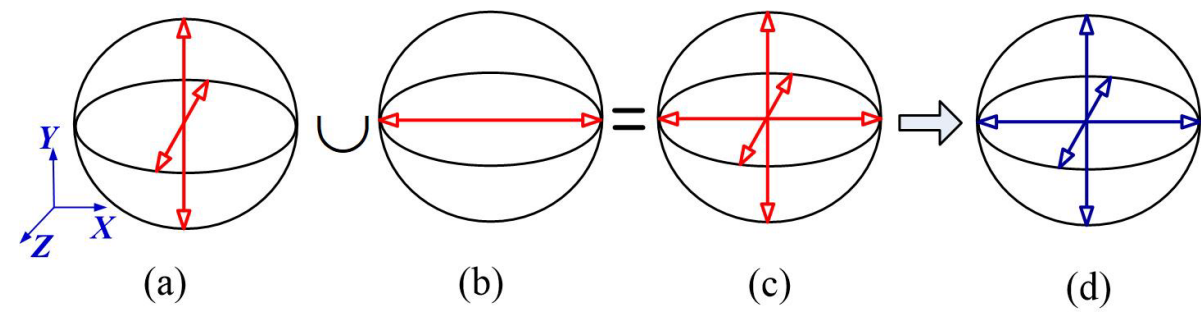

Figure 5. Constraint and DoF space graph of the whole manipulator: (a) PLMEs limb, (b) Single Loop 2-UPR, (c) Constraint space of moving platform, (d) DoF space of moving platform.

used in the research on mechanism, especially on the DoFs and constraints of a mechanism. The line graph can be used to express an $n$-dimensional DoF space or constraint space in a mechanism. Blanding proposed a basic rule to uncover this dual relationship and mutual converse of the DoF spaces and constraint spaces through line graph. The basic rule was summarized by Blanding (1999); Yu et al. (2011), and etc. In this paper, we firstly plot the constraint space of the PLMEs limb and the single loop 2-UPR in one graph with Grassmann line geometry, as shown in Fig. 5c. Secondly, we apply the mutual conversion rule of DoF spaces and constraint spaces (Xie et al., 2013) for Fig. 5c. Then, the DoF space can be quickly obtained as shown in Fig. 5d. The result illustrates that the platform has 3 translational DoFs.

The manipulator has 2 kinds of singularities. One kind of singularity comes from the PLMEs limb. When $\theta_{1}= \pm \pi / 2$ or $\theta_{1}=0, \pi$, the PLMEs limb is located on singular Plane $\eta_{1}$ or Line $\varsigma_{1}$, as shown in Fig. 1. In this case, the constraints of the PLMEs limb are instantaneously changed. It results into the varying of the mobility of the manipulator. Another kind of singularity is that the 2-UPR limb are vertical, located on Plane $\eta_{2}$. In this case, the constraints of the PLMEs limb and 2-URP limbs are all on $Y-Z$ plane. The $X$ axis rotational constraint is absent. The moving platform exists an extra instantaneous rotation about $X$ axis.

In this manipulator, each limb is assumed to be driven by one linear actuator. Herein, we discuss whether the moving platform can be controlled by these 3 selected linear actuators. For the convenience of calculation, the moving platform is regarded as a link, without consideration of the actual geometric feature. Thus, the coordinates of the joints in the moving platform yield

$x_{e_{2}}=x_{c_{2}}=x_{c_{1}}, \quad y_{e_{2}}=y_{c_{2}}=y_{c_{1}}$

In analysis, the two prismatic joints in the single loop 2UPR are firstly fixed. The prismatic joint in PLMEs limb is free. In this case, the twist $\$_{A_{1}}$ of the moving platform can be obtained as

$\$_{A_{1}}=\left[\begin{array}{llllll}0, & 0, & 0, & -\left(y_{a_{1}}-y_{c_{1}}\right), \quad\left(x_{a_{1}}-x_{c_{1}}\right), & 0\end{array}\right]$

Equation (11) illustrates that the moving platform has only one translational mobility under the above condition. The in- stantaneous velocity is perpendicular with the plane formed by $D_{2} Q_{2}$ and $D_{3} Q_{3}$.

Secondly, the prismatic joints in PLMEs and Limb $D_{3} Q_{3}$ are fixed and the prismatic joint in Limb $D_{2} Q_{2}$ is set to be free. The twist $\$_{A_{2}}$ of the moving platform can be calculated as

$\$_{A_{2}}=\left[\begin{array}{l}0, \quad 0, \quad 0, \quad\left(z_{a_{2}}-z_{c_{2}}\right) \sin \theta_{1}, \\ -\left(z_{a_{2}}-z_{c_{2}}\right) \cos \theta_{1}, \\ \left(y_{a_{2}}-y_{c_{2}}\right) \cos \theta_{1}-\left(x_{a_{2}}-x_{c_{2}}\right) \sin \theta_{1}\end{array}\right]$

Equation (12) illustrates that the moving platform has one translational mobility, which is perpendicular with the plane formed by $D_{1} E_{1}$ and $D_{3} Q_{3}$.

In the same way, the prismatic joints in PLMEs and Limb $D_{2} Q_{2}$ are fixed and the prismatic joint in Limb $D_{3} Q_{3}$ is set to be free. The twist $\$_{A_{3}}$ of the moving platform is

$\$_{A_{3}}=\left[\begin{array}{l}0, \quad 0, \quad 0, \quad\left(z_{a_{1}}-z_{c_{1}}\right) \sin \theta_{1}, \\ -\left(z_{a_{1}}-z_{c_{1}}\right) \cos \theta_{1}, \\ \left(y_{a_{1}}-y_{c_{1}}\right) \cos \theta_{1}-\left(x_{a_{1}}-x_{c_{1}}\right) \sin \theta_{1}\end{array}\right]$

Equation (13) illustrates that the only one translational mobility is perpendicular with the plane formed by $D_{1} E_{1}$ and $D_{2} Q_{2}$.

According to the above analysis, it can be summarized that the manipulator assembled by PLMEs and 2-UPR limbs generally has 3 pure translational DoFs. And the moving platform is controllable by 3 linear actuators.

\section{Simplified kinematic and dynamic model}

According to the motion characteristics of this manipulator, the 3-DoF translational manipulator can be simplified as a 3-SPS mechanism without rotation mobilities. As shown in Fig. 6, $\left(x_{C_{1}}, y_{C_{1}}, z_{C_{1}}\right),\left(x_{C_{2}}, y_{C_{2}}, z_{C_{2}}\right)$ and $\left(x_{C_{3}}, y_{C_{3}}, z_{C_{3}}\right)$ are the initial positions of the points $C_{1}, C_{2}$ and $C_{3}$ of the moving platform. $\left(x_{\mathrm{A} 1}, y_{\mathrm{A} 1}, z_{A_{1}}\right),\left(x_{A_{2}}, y_{A_{2}}, z_{A_{2}}\right),\left(x_{A_{3}}, y_{A_{3}}, z_{A_{3}}\right)$ are the initial positions of the points $A_{1}, A_{2}$ and $A_{3}$ of the fixed platform, respectively. $l_{1}, l_{2}$ and $l_{3}$ are the lengths of $A_{1} C_{1}, A_{2} C_{2}$ and $A_{3} C_{3}$, which can be regarded as the linear actuators of the manipulator. 


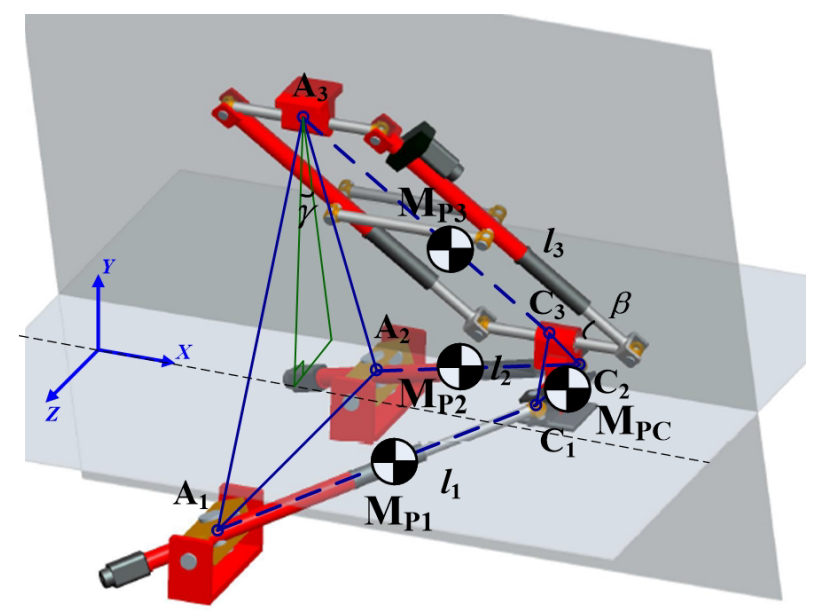

Figure 6. Simplified 3-SPS mechanism.

\subsection{Displacement equation}

Let $x, y, z$ be the displacements of the moving platform $C_{1} C_{2} C_{3}$ from the initial position. The displacement equations can be derived as

$\left\{\begin{array}{l}\sqrt{\left(x_{C_{1}}+x-x_{A_{1}}\right)^{2}+\left(y_{C_{1}}+y-y_{A_{1}}\right)^{2}+\left(z_{C_{1}}+z-z_{A_{1}}\right)^{2}}=l_{1} \\ \sqrt{\left(x_{C_{2}}+x-x_{A_{2}}\right)^{2}+\left(y_{C_{2}}+y-y_{A_{2}}\right)^{2}+\left(z_{C_{2}}+z-z_{A_{2}}\right)^{2}}=l_{2} \\ \sqrt{\left(x_{C_{3}}+x-x_{A_{3}}\right)^{2}+\left(y_{C_{3}}+y-y_{A_{3}}\right)^{2}+\left(z_{C_{3}}+z-z_{A_{3}}\right)^{2}}=l_{3}\end{array}\right.$

If the displacement $(x, y, z)$ is known, the driving displacements of the linear actuators $l_{1}, l_{2}$ and $l_{3}$ can be easily obtained by Eq. (14). Conversely, if the driving displacements $l_{1}, l_{2}$ and $l_{3}$ are known, the forward displacement of the moving platform $(x, y, z)$ can be found by solving the above 3 equations. Let

$X_{A_{1}}=x_{A_{1}}-x_{\mathrm{C} 1}, \quad X_{A_{2}}=x_{A_{2}}-x_{C_{2}}, \quad X_{A_{3}}=x_{A_{3}}-x_{C_{3}}$,

$Y_{A_{1}}=y_{A_{1}}-y_{\mathrm{C} 1}, \quad Y_{A_{2}}=y_{A_{2}}-y_{C_{2}}, \quad Y_{A_{3}}=y_{A_{3}}-y_{C_{3}}$,

$Z_{A_{1}}=z_{A_{1}}-z_{\mathrm{C} 1}, \quad Z_{A_{2}}=z_{A_{2}}-z_{C_{2}}, \quad Z_{A_{3}}=z_{A_{3}}-z_{C_{3}}$

and

$E_{11}=2\left(X_{A_{2}}-X_{A_{1}}\right), \quad E_{12}=2\left(Y_{A_{2}}-Y_{A_{1}}\right)$,

$E_{13}=2\left(Z_{A_{2}}-Z_{A_{1}}\right), \quad E_{31}=2\left(X_{A_{3}}-X_{A_{2}}\right)$,

$E_{32}=2\left(Y_{A_{3}}-Y_{A_{2}}\right), \quad E_{33}=2\left(Z_{A_{3}}-Z_{A_{2}}\right)$

$H_{1}=l_{1}^{2}-l_{2}^{2}+\left(X_{A_{2}}^{2}-X_{A_{1}}^{2}\right)+\left(Y_{A_{2}}^{2}-Y_{A_{1}}^{2}\right)+\left(Z_{A_{2}}^{2}-Z_{A_{1}}^{2}\right)$

$H_{3}=l_{2}^{2}-l_{3}^{2}+\left(X_{A_{3}}^{2}-X_{A_{2}}^{2}\right)+\left(Y_{A_{3}}^{2}-Y_{A_{2}}^{2}\right)+\left(Z_{A_{3}}^{2}-Z_{A_{2}}^{2}\right)$

Squaring both sides of Eq. (14), and subtracting the first formula to the second one, and the second one to the third one, Eq. (14) is transformed to

$\left\{\begin{array}{l}E_{11} x+E_{12} y+E_{13 z}=H_{1} \\ E_{31} x+E_{32} y+E_{33} z=H_{3}\end{array}\right.$
In this paper, the limbs $A_{1} A_{2}$ and $C_{1} C_{2}$ are always perpendicular with $X-Y$ plane. The length projections of $A_{1} C_{1}$ and $A_{2} C_{2}$ on $X-Y$ plane are equal. It yields

$E_{11}=E_{12}=0$

Through Eqs. (15) and (16), the displacement $z$ can be quickly solved

$z=H_{1} / E_{13}$

Then, substituting Eq. (17) into Eqs. (14) and (15), and let

$m=-E_{32} / E_{31}, n=\left(H_{3}-E_{33} H_{1} / E_{13}\right) / E_{31}$,

$P_{a}=m^{2}+1, \quad P_{b}=2\left(m\left(n-X_{A_{3}}\right)-Y_{A_{3}}\right)$,

$P_{c}=\left(n-X_{A_{3}}\right)^{2}+Y_{A_{3}}{ }^{2}+\left(z-Z_{A_{3}}\right)^{2}-l_{3}^{2}$

The displacements $y$ and $x$ can be solved by

$\left\{\begin{array}{l}y=\frac{-P_{b}-\sqrt{P_{b}^{2}-4 P_{a} P_{c}}}{2 P_{a}} \\ x=m y+n\end{array}\right.$

Equation (17) and (18) give the analytic solution of the forward kinematics of the manipulator. Further, we can calculate the angle $\beta$ (as shown in Fig. 6) in the PLMEs limb by the following formula.

$\cos \beta=$

$-\frac{x_{C_{1}}+x-x_{A_{1}}}{\sqrt{\left(x_{C_{1}}+x-x_{A_{1}}\right)^{2}+\left(y_{C_{1}}+y-y_{A_{1}}\right)^{2}+\left(z_{C_{1}}+z-z_{A_{1}}\right)^{2}}}$

And the angle $\gamma$ between the PLMEs and the horizontal plane (as shown in Fig. 6) is calculated as

$\tan \gamma=\frac{z_{C_{1}}+z-z_{A_{1}}}{y_{C_{1}}+y-y_{A_{1}}}$

\subsection{Velocity equation}

Differentiating Eq. (14) leads to the velocity equation.

$\mathbf{J}_{v}\left[\begin{array}{c}\dot{x} \\ \dot{y} \\ \dot{z}\end{array}\right]=\left[\begin{array}{l}i_{1} \\ \dot{l}_{2} \\ \dot{l}_{3}\end{array}\right]$

where the Jacobian matrix $\mathbf{J}_{v}$ is

$\mathbf{J}_{v}=$

$\left[\begin{array}{lll}\frac{\left(x-x_{A_{1}}+x_{C_{1}}\right)}{l_{1}} & \frac{\left(y-y_{A_{1}}+y_{C_{1}}\right)}{l_{1}} & \frac{\left(z-z_{A_{1}}+z_{C_{1}}\right)}{l_{1}} \\ \frac{\left(x-x_{A_{2}}+x_{C_{2}}\right)}{l_{2}} & \frac{\left(y-y_{A_{2}}+y_{C_{2}}\right)}{l_{2}} & \frac{\left(z-z_{A_{2}}+z_{C_{2}}\right)}{l_{2}} \\ \frac{\left(x-x_{A_{3}}+x_{C_{3}}\right)}{l_{3}} & \frac{\left(y-y_{A_{3}}+y_{C_{3}}\right)}{l_{3}} & \frac{\left(z-z_{A_{3}}+z_{C_{3}}\right)}{l_{3}}\end{array}\right]$

In Eq. (22), each row of the matrix $\mathbf{J}_{v}$ represents the cosines of the angles between the corresponding SPS limb and the $x$, 
$y$, and $z$ axes. The absolute value of the determinant of the third-order matrix $\mathbf{J}_{v}$ equals the volume of the parallelepiped spanned by each row vector of $\mathbf{J}_{v}$. If the 3 SPS limbs are collinear or coplanar, the determinant of the Jacobian matrix equals zero. This mechanism is in singular configuration, which should be avoided in the motion planning. If an external force $\left(F_{x}, F_{y}, F_{z}\right)$ was exerted on the moving platform, the static driving forces of the linear actuators $\left(f_{1}, f_{2}, f_{3}\right)$ corresponding to limbs 1, 2 and 3 could be obtained through the Jacobian matrix.

$$
\left[\begin{array}{l}
F_{x} \\
F_{y} \\
F_{z}
\end{array}\right]=\mathbf{J}_{v}^{\mathrm{T}}\left[\begin{array}{l}
f_{1} \\
f_{2} \\
f_{3}
\end{array}\right]
$$

\subsection{Dynamic equation}

In the manipulator, the moving platform is assumed to carry a heavy load. Taking the mass of 3 limbs as $M_{P_{1}}, M_{P_{2}}$ and $M_{P_{3}}$, each limb of the manipulator is simplified into a mass point for convenient calculation, which is located in the center of the limb as shown in Fig. 6. $M_{\mathrm{PC}}$ is the mass of the moving platform with the heavy load. Based on these assumptions, the simplified dynamics equation can be written as

$$
\begin{aligned}
& {\left[\begin{array}{c}
f_{1} \\
f_{2} \\
f_{3}
\end{array}\right]=} \\
& \left(\mathbf{J}_{v}^{\mathrm{T}}\right)^{-1}\left(-M_{\mathrm{PC}} \mathbf{I}_{3 \times 3}\left(\begin{array}{c}
\ddot{x} \\
\ddot{y} \\
\ddot{z}
\end{array}\right)-\frac{1}{2} \sum_{i=1}^{3} M_{P_{i}} \mathbf{I}_{3 \times 3}\left(\begin{array}{c}
\ddot{x} \\
\ddot{y} \\
\ddot{z}
\end{array}\right)\right. \\
& +M_{\mathrm{PC}} \mathbf{I}_{3 \times 3}\left(\begin{array}{c}
g_{x} \\
g_{y} \\
g_{z}
\end{array}\right)+\frac{1}{2} \sum_{i=1}^{3} M_{P_{i}} \mathbf{I}_{3 \times 3}\left(\begin{array}{c}
g_{x} \\
g_{y} \\
g_{z}
\end{array}\right) \\
& \left.+\left(\begin{array}{c}
F_{x} \\
F_{y} \\
F_{z}
\end{array}\right)\right)
\end{aligned}
$$

where $\mathbf{I}_{3 \times 3}$ is the $3 \times 3$ identity matrix. $F_{x}, F_{y}$ and $F_{z}$ are the external force exerted on the moving platform. $g_{x}, g_{y}$ and $g_{z}$ are the gravitational acceleration. And $f_{i}(i=1,2,3)$ are the driving forces of the 3 linear actuators. Let

$\mathbf{M}_{P}=M_{\mathrm{PC}} \mathbf{I}_{3 \times 3}+\frac{1}{2} \sum_{i=1}^{3} M_{P_{i}} \mathbf{I}_{3 \times 3}$

Assume $\dot{l}_{i}$ and $\ddot{l}_{i}(i=1,2,3)$ be the velocity and acceleration of the actuators. Differentiating Eq. (21) and substituting the result into Eq. (24), it leads to

$$
\left[\begin{array}{l}
f_{1} \\
f_{2} \\
f_{3}
\end{array}\right]=
$$

$$
\begin{aligned}
& -\left(\mathbf{J}_{v}^{\mathrm{T}}\right)^{-1} \mathbf{M}_{P} \mathbf{J}_{v}^{-1}\left[\begin{array}{l}
\ddot{l}_{1} \\
\ddot{l}_{2} \\
\ddot{l}_{3}
\end{array}\right]-\left(\mathbf{J}_{v}^{\mathrm{T}}\right)^{-1} \mathbf{M}_{P} \mathbf{J}_{v}^{-1}\left[\begin{array}{l}
\dot{l}_{1} \\
\dot{l}_{2} \\
\dot{l}_{3}
\end{array}\right] \\
& +\left(\mathbf{J}_{v}^{\mathrm{T}}\right)^{-1} \mathbf{M}_{P}\left[\begin{array}{l}
g_{x} \\
g_{y} \\
g_{z}
\end{array}\right] \\
& +\left(\mathbf{J}_{v}^{\mathrm{T}}\right)^{-1}\left(\begin{array}{l}
F_{x} \\
F_{y} \\
F_{z}
\end{array}\right)
\end{aligned}
$$

Considering

$\dot{\mathbf{J}}_{v}^{-1}=-\mathbf{J}_{v}^{-1} \dot{\mathbf{J}}_{v} \mathbf{J}_{v}^{-1}$

and substituting the above equation into Eq. (26), the equation can be rewritten as

$$
\begin{aligned}
& {\left[\begin{array}{c}
f_{1} \\
f_{2} \\
f_{3}
\end{array}\right]=} \\
& -\left(\mathbf{J}_{v}^{\mathrm{T}}\right)^{-1} \mathbf{M}_{P} \mathbf{J}_{v}^{-1}\left(\begin{array}{c}
\ddot{l}_{1} \\
\ddot{l}_{2} \\
\ddot{l}_{3}
\end{array}\right)+\left(\mathbf{J}_{v}^{\mathrm{T}}\right)^{-1} \mathbf{M}_{P} \mathbf{J}_{v}^{-1} \mathbf{J}_{v} \mathbf{J}_{v}^{-1}\left(\begin{array}{c}
\dot{l}_{1} \\
\dot{i}_{2} \\
\ddot{l}_{3}
\end{array}\right) \\
& +\left(\mathbf{J}_{v}^{\mathrm{T}}\right)^{-1} \mathbf{M}_{P}\left(\begin{array}{c}
g_{x} \\
g_{y} \\
g_{z}
\end{array}\right)+\left(\mathbf{J}_{v}^{\mathrm{T}}\right)^{-1}\left(\begin{array}{c}
F_{x} \\
F_{y} \\
F_{z}
\end{array}\right)
\end{aligned}
$$

where the derivative of Jacobian matrix $\dot{\mathbf{J}}_{v}$ is

$$
\begin{aligned}
& \dot{\mathbf{J}}_{v}=\left[\begin{array}{l}
\frac{\dot{x} l_{1}-\left(\frac{\partial l_{1}}{\partial x} \dot{x}+\frac{\partial l_{1}}{\partial y} \dot{y}+\frac{\partial l_{1}}{\partial z} \dot{z}\right)\left(x-X_{A_{1}}\right)}{l_{1}^{2}} \\
\frac{\dot{x} l_{2}-\left(\frac{\partial l_{2}}{\partial x} \dot{x}+\frac{\partial l_{2}}{\partial y} \dot{y}+\frac{\partial l_{2}}{\partial z} \dot{z}\right)\left(x-X_{A_{2}}\right)}{l_{2}^{2}} \\
\frac{\dot{x} l_{3}-\left(\frac{\partial l_{3}}{\partial x} \dot{x}+\frac{\partial l_{3}}{\partial y} \dot{y}+\frac{\partial l_{3}}{\partial z} \dot{z}\right)\left(x-X_{A_{3}}\right)}{l_{3}^{2}}
\end{array}\right. \\
& \frac{\dot{y} l_{1}-\left(\frac{\partial l_{1}}{\partial x} \dot{x}+\frac{\partial l_{1}}{\partial y} \dot{y}+\frac{\partial l_{1}}{\partial z} \dot{z}\right)\left(y-Y_{A_{1}}\right)}{l_{1}^{2}} \\
& \frac{\dot{y} l_{2}-\left(\frac{\partial l_{2}}{\partial x} \dot{x}+\frac{\partial l_{2}}{\partial y} \dot{y}+\frac{\partial l_{2}}{\partial z} \dot{z}\right)\left(y-Y_{A_{2}}\right)}{l_{2}^{2}} \\
& \frac{\dot{y} l_{3}-\left(\frac{\partial l_{3}}{\partial x} \dot{x}+\frac{\partial l_{3}}{\partial y} \dot{y}+\frac{\partial l_{3}}{\partial z} \dot{z}\right)\left(y-Y_{A_{3}}\right)}{l_{3}^{2}} \\
& \left.\frac{\dot{z} l_{1}-\left(\frac{\partial l_{1}}{\partial x} \dot{x}+\frac{\partial l_{1}}{\partial y} \dot{y}+\frac{\partial l_{1}}{\partial z} \dot{z}\right)\left(z-Z_{A_{1}}\right)}{l_{1}^{2}}\right] \\
& \frac{\dot{z} l_{2}-\left(\frac{\partial l_{2}}{\partial x} \dot{x}+\frac{\partial l_{2}}{\partial y} \dot{y}+\frac{\partial l_{2}}{\partial z} \dot{z}\right)\left(z-Z_{A_{2}}\right)}{l_{2}^{2}} \\
& \left.\frac{\dot{z} l_{3}-\left(\frac{\partial l_{3}}{\partial x} \dot{x}+\frac{\partial l_{3}}{\partial y} \dot{y}+\frac{\partial l_{3}}{\partial z} \dot{z}\right)\left(z-Z_{A_{3}}\right)}{l_{3}^{2}}\right]
\end{aligned}
$$




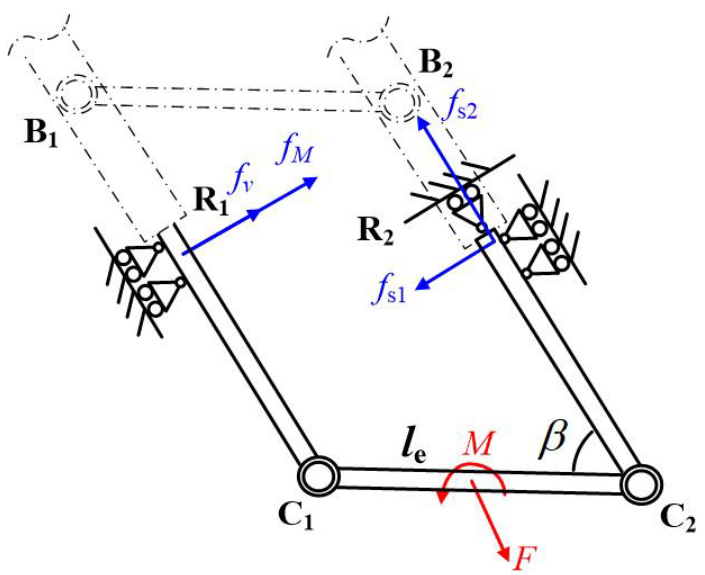

Figure 7. Reaction forces of the prismatic joints in the planar PLMEs limb

Eq. (28) provides a simplified dynamic model for this translational parallel manipulator. It could be used into the control strategy, especially in the high speed pick-and-place operation.

\section{Reaction forces of the prismatic joints in the PLMEs limb}

In this manipulator, the PLMEs limb provides 2 rotational constraints to the moving platform. The varying of the reaction forces of the two prismatic joints in the PLMEs limb could reveal the performance of the manipulator, i.e., singularity, stiffness, etc. Thus, we mainly concern the reaction forces of the prismatic joints in the PLMEs limb in this section. We firstly study the reaction forces of the prismatic joints in the $X-Y$ plane. Secondly, an external force is exerted on the moving platform. And then the reaction forces of the prismatic joints are investigated in the context of the whole manipulator. The results provide the basis for the design of the PLMEs limb in this new translational parallel manipulator.

\subsection{Reaction forces in the planar PLMEs limb}

In the beginning, we only take the PLMEs limb into the analysis in the $X-Y$ plane, without consideration of the Loop 2UPR. As shown in Fig. 7, $B_{1} C_{1}$ and $B_{2} C_{2}$ are parallel. $R_{1}$ and $R_{2}$ are the two prismatic joints. Assumed the actuator is located on $R_{2}, B_{2} C_{2}$ can be regarded to fix on the ground. An external force $F$ and an external torque $M$ are exerted on the middle of Link $C_{1} C_{2}$, as shown in Fig. 7. The length of Link $C_{1} C_{2}$ is $l_{e}$. The orientation of $F$ is along with the PLMEs limb.

Taking $f_{v}$ as the reaction force corresponding to the force $F$, and $f_{M}$ as the reaction force corresponding to the torque $M$, the following equations are established by means of the virtual work principle.

$$
\left\{\begin{array}{l}
F \cdot \delta\left(\frac{l_{e}}{2} \cos \beta\right)=-f_{v} \cdot \delta\left(l_{e} \sin \beta\right) \\
f_{M} \cdot \delta\left(l_{e} \sin \beta\right)=M \cdot \delta \beta
\end{array}\right.
$$

Solving Eq. (30), we can obtain

$$
\left\{\begin{array}{c}
f_{v}=\frac{F}{2} \tan \beta \\
f_{M}=\frac{M}{l_{e} \cos \beta}
\end{array}\right.
$$

By the sum of $f_{v}$ and $f_{M}$, the total reaction force $f_{R_{1}}$ of the prismatic joint $R_{1}$ can be calculated as the following equation. The orientation of $f_{R_{1}}$ is perpendicular with the prismatic joint.

$$
f_{R_{1}}=f_{v}+f_{M}=\frac{F}{2} \tan \beta+\frac{M}{l_{e} \cos \beta}
$$

The reaction force $f_{R_{2}}$ of the other prismatic joint $R_{2}$ is decomposed into 2 directions. One direction force $f_{s_{1}}$ is perpendicular with Line $B_{2} C_{2}$ and opposite to $f_{R_{1}}$. The other direction force $f_{s_{2}}$ is along with Line $B_{2} C_{2}$ and opposite to the actuator force $F$. Hence, the component forces $f_{s_{1}}$ and $f_{S_{2}}$ are written as

$\left\{\begin{array}{l}f_{s_{1}}=-f_{R_{1}} \\ f_{s_{2}}=-F\end{array}\right.$

Observing Eqs. (32) and (33), it is noticed that $f_{R_{1}}$ and $f_{s_{1}}$ becomes infinite if $\beta=\pi / 2$. It indicates that the PLMEs limb with $\beta=\pi / 2$ is in the singular configuration, which is consistent with the result derived in Sect. 2.

\subsection{Reaction forces in the context of the whole manipulator}

Based on the above results, we furthermore investigate the reaction forces of the PLMEs limb in the context of the whole manipulator. The moving platform bears a vertical force $F_{G}$ as shown in Fig. 8a. Through Eq. (23), the 3 linear actuator forces, denoted as $F_{A_{1}}, F_{A_{2}}$ and $F_{A_{3}}$, could be firstly calculated out. Secondly, by the utilization of $F_{A_{3}}$ and the first row of Eq. (31), one component reaction force $f_{G_{1}}$ of the prismatic joints, which is directly corresponding to the force $F_{A_{3}}$, can be obtained as following.

$f_{G_{1}}=\frac{F_{A_{3}}}{2} \tan \beta$

As shown in Fig. 8a, there is a "T" shaped conjunction part connecting the PLMEs limb and the 2-UPR limb. If there is a small distance $e$ between the two connections in the " $T$ " conjunction part, the resultant force leads to a torque $M_{h}$ exerted 


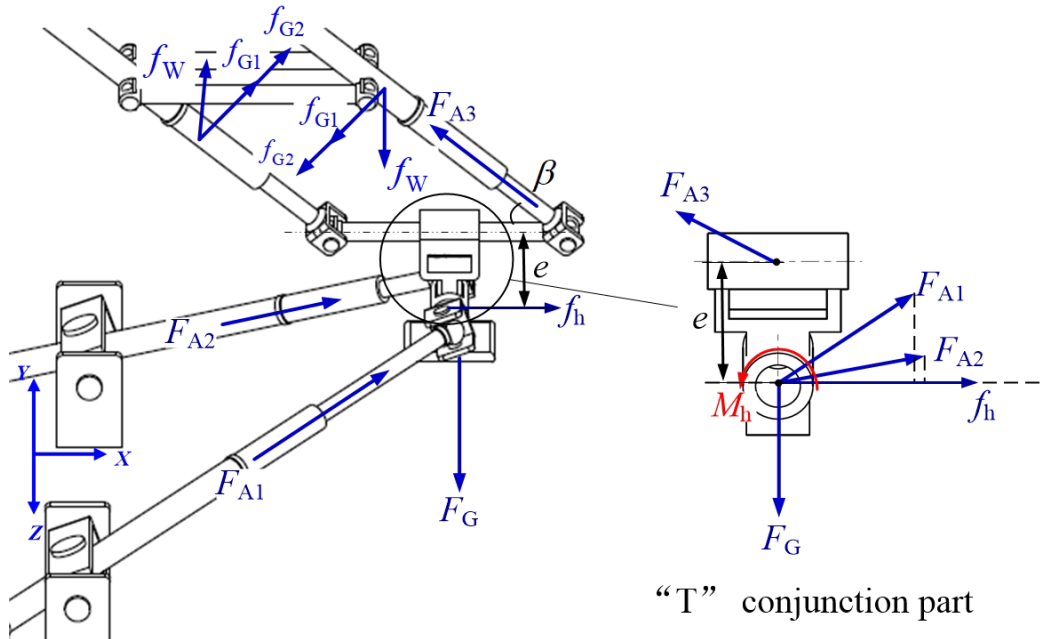

(a)

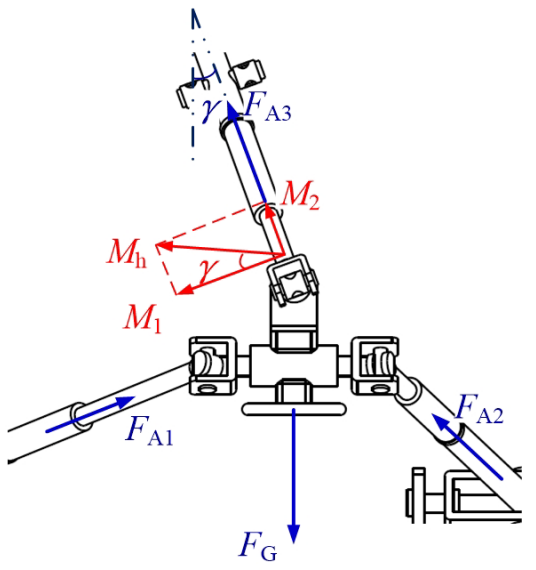

(b)

Figure 8. Reaction forces of the prismatic joints in the context of the whole manipulator: (a) side view, (b) front view.

on the "T" conjunction part. The torque $M_{h}$ can be obtained by

$M_{h}=f_{h} \cdot e$

where $f_{h}$ is the resultant force of $F_{A_{1}}$ and $F_{A_{2}}$ projected on $X$ axis. As shown in Fig. $8 \mathrm{~b}, M_{h}$ can be decomposed into $M_{1}$ and $M_{2}$ by Eq. (36).

$M_{1}=M_{h} \cos \gamma, M_{2}=M_{h} \sin \gamma$

where $M_{1}$ and $M_{2}$ represent the torques be in and perpendicular to the plane of PLMEs, respectively. The angle $\gamma$ between the PLMEs and the horizontal plane can be found by Eq. (20). According to the second row of Eq. (31), the reaction force $f_{G_{2}}$ corresponding to the torque $M_{1}$ can be written as

$f_{G_{2}}=\frac{M_{1}}{l_{e} \cos \beta}$

Thus, the total reaction force $f_{N}$ perpendicular to the prismatic joint in the plane of PLMEs can be obtained by sum$\operatorname{ming} f_{G_{1}}$ and $f_{G_{2}}$.

$f_{N}=f_{G_{1}}+f_{G_{2}}=\frac{F_{A_{3}}}{2} \tan \beta+\frac{M_{1}}{l_{e} \cos \beta}$

In another way, the torque $M_{2}$ would cause the other reaction force $f_{W}$ perpendicular to the plane of PLMEs. It can be figured out by Eq. (39).

$f_{W}=\frac{M_{2}}{l_{e}}$

Finally, through $f_{N}$ and $f_{W}$, the resultant reaction force $f_{\mathrm{c}}$ of the prismatic joint can be obtained as follows

$f_{c}=\sqrt{f_{N}^{2}+f_{W}^{2}}$
All the above calculation procedure for the force of PLMEs limb is summarized in Fig. 9. It shows that the resultant reaction force of the prismatic joints is related to the Jacobian matrix of the manipulator and the configuration of the PLMEs limb. If the reaction force becomes large, it indicates the manipulator is in bad performance(e.g., in case of singularity), and vice versa. Through analyzing the force of the prismatic joints in the PLMEs limb, the performance of the manipulator can be revealed.

Based on the above-mentioned results, it is also found that the way of making the external load $F_{G}$ be closed to the actuator of PLMEs limb, or decreasing the distance $e$ between PLMEs limb and 2-UPR limb could reduce the resultant reaction force of the prismatic joints. It is very helpful for the design of this new translational parallel manipulator.

\section{Performance investigation}

Based on the aforementioned analysis, we discuss the workspace, condition numbers of Jacobian matrices, simplified dynamics and motion transmission of the new manipulator. And the reaction forces of the manipulator under the circumstance that the moving platform bearing a vertical force is also investigated.

\subsection{Workspace and Jacobian matrices}

We assume that the ranges of 3 linear actuators are [-300, 300], [-300, 300] and [-210, 210] for Limbs $L_{1}, L_{2}$ and $L_{3}$ relative to the initial configuration. The joint positions of the initial configuration are listed in Table 1. 
Table 1. Coordinates of the initial points $C_{i}(i=1,2,3)$ and $A_{i}(i=1,2,3)$.

\begin{tabular}{lccc}
\hline Initial points & 1 & 2 & 3 \\
\hline$\left(x_{A_{i}}, y_{A_{i}}, z_{A_{i}}\right)$ & $(-541.3,-36.0,634.0)$ & $(-541.3,-36.0,-634.0)$ & $(-429.5,441.0,0.0)$ \\
$\left(x_{C_{i}}, y_{C_{i}}, z_{C_{i}}\right)$ & $(0.0,0.0,91.8)$ & $(0.0,0.0,-91.8)$ & $(0.0,79.2,0.0)$ \\
\hline
\end{tabular}

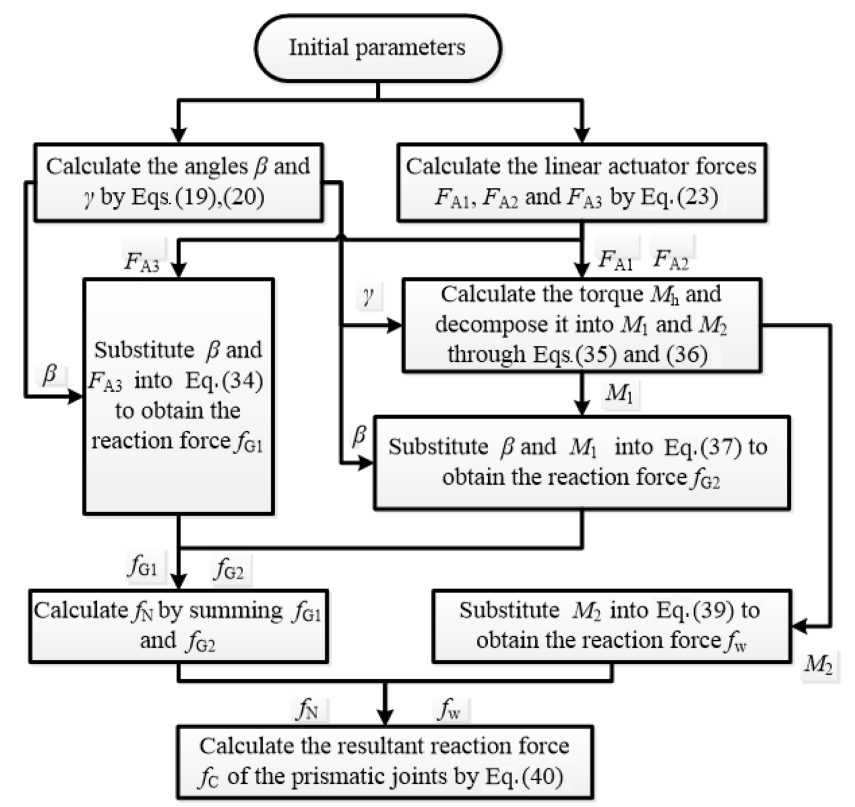

Figure 9. Calculation procedure for the reaction forces of the prismatic joints.

Considering the initial configuration, the absolute motion range $\left[l_{\min }, l_{\max }\right]$ of each limb is $[467.0,1067.0]$, [467.0, 1067.0] and [351.6, 771.6], respectively. According to Eq. (14), the workspace of the moving platform is the intersection of the 3 hollow spheres, whose inner and outer radii are $l_{\min }$ and $l_{\max }$. And the centers of the 3 spheres are respectively at $\left(X_{A_{1}}, Y_{A_{1}}, Z_{A_{1}}\right),\left(X_{A_{2}}, Y_{A_{2}}, Z_{A_{2}}\right)$ and $\left(X_{A_{3}}, Y_{A_{3}}, Z_{A_{3}}\right)$. To avoid singularity and consider the actual usage, we just calculate the workspace in the range of $y \leq 441.0$ and $x \geq-429.5$, as shown in Fig. 10a. The workspace of the moving platform is plotted as shown in Fig. 10b-d. The volume of the workspace is calculated to be $1.9755 \times 10^{8} \mathrm{~mm}^{3}$.

Furthermore, the condition numbers of Jacobian matrices in the workspace is figured out to evaluate the kinematic performance of the manipulator. In Fig. 11, the condition numbers are plotted as the contour lines on the different layers which are respectively located on the planes $y=-400,-300,-200,-100,0,100,200,300$ and the plane $z=0$. The contour lines show that the condition numbers are large when the manipulator approaches the singular configurations, i.e., $\beta=0$ and $\beta=\pi / 2$. It illustrates that the kinematic property becomes bad when the moving platform is in these areas. In the proceeding of the motion planning, it is better to avoid these areas.

\subsection{Simplified dynamics}

In the dynamic analysis, the joint positions of the initial configuration are as the same as listed in Table 1. The mass of Limbs 1, 2 and 3 are 2.676, 2.676 and $5.35 \mathrm{~kg}$, respectively. And the mass of the moving platform with the load is $11.237 \mathrm{~kg}$. An external force $F_{y}=-100 \mathrm{~N}$ is exerted on the moving platform. The gravity is along the negative $Y$ axis. We give the displacement equation $\mathrm{d} l_{i}$ of each linear actuator relative to the initial configuration as follows.

$\mathrm{d} l_{i}=140 \sin \left(\frac{\pi}{4} t+\frac{2 i}{3} \pi\right) \quad i=1,2,3$

Adding the initial length of each limb $l_{o_{i}}$, the absolute displacement equation of each limb is obtained as

$l_{i}=l_{o_{i}}+\mathrm{d} l_{i} \quad i=1,2,3$

Substituting the above conditions into Eq. (28), the actuating force of each limb can be calculated out. To validate the effectiveness of the simplified model deduced in Sect. 3.3, the complete dynamic model is built and simulated in ADAMS software. Two results are presented in Fig. 12, where the solid lines represent the ones come from the simplified dynamic model, the dashed lines represent the ones come from ADAMS software. It shows that the two curves are generally consistent with each other, although some local errors are a bit of large. The errors come from the simplification of each limb into the mass point. It results in the decreasing of the model accuracy. Nevertheless, the simplified dynamic model still make sense to estimate the actuating force or make control strategy in the design of this manipulator.

Furthermore, the reaction forces of the prismatic joints are also calculated based on the method proposed in Sect. 4.2. The results are compared with the ones obtained by ADAMS software, as shown in Fig. 13. It is found that the two results are similar, which proves the correctness of the method proposed in Sect. 4.2.

\subsection{Motion transmission}

To evaluate the efficiency of the power transmission of the manipulator, the motion transmission indices of each leg are studied by screw theory (Wu et al., 2010; Zhao et al., 2017). For a given configuration, the transmission wrench 


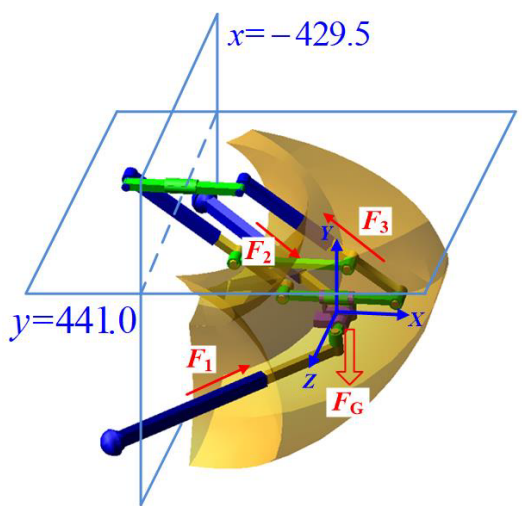

(a)

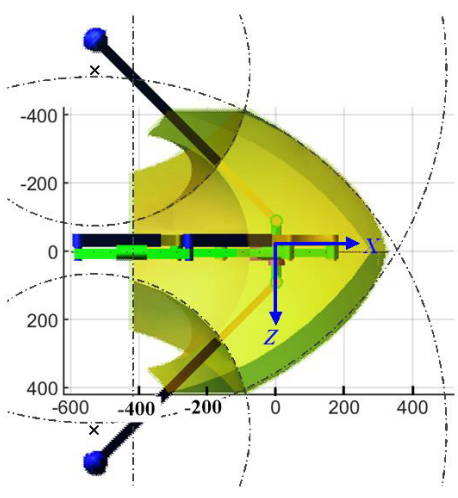

(c)

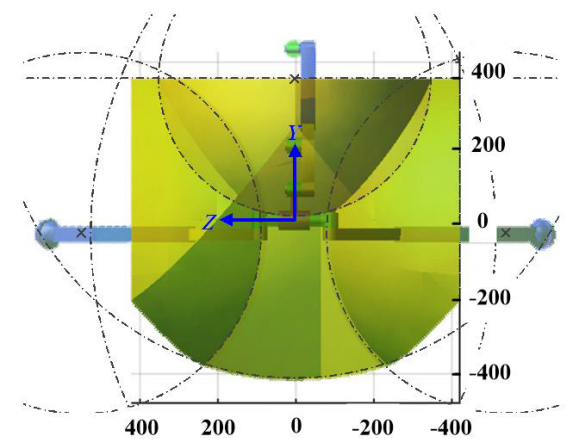

(b)

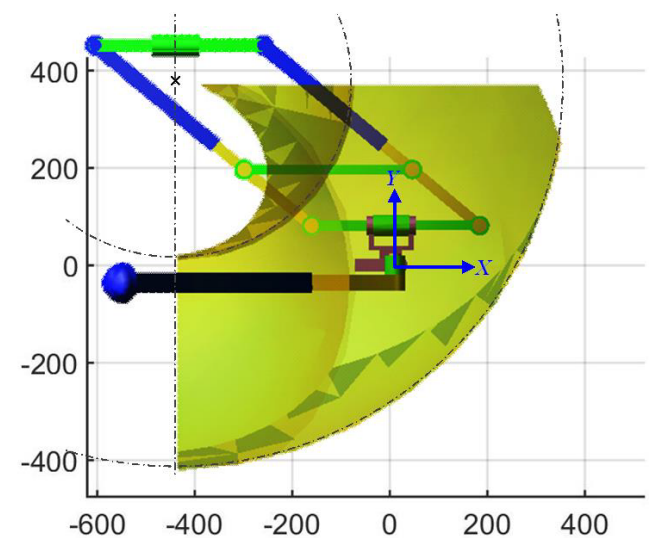

(d)

Figure 10. Workspace of the moving platform: (a) oblique view, (b) front view, (c) top view, (d) side view.

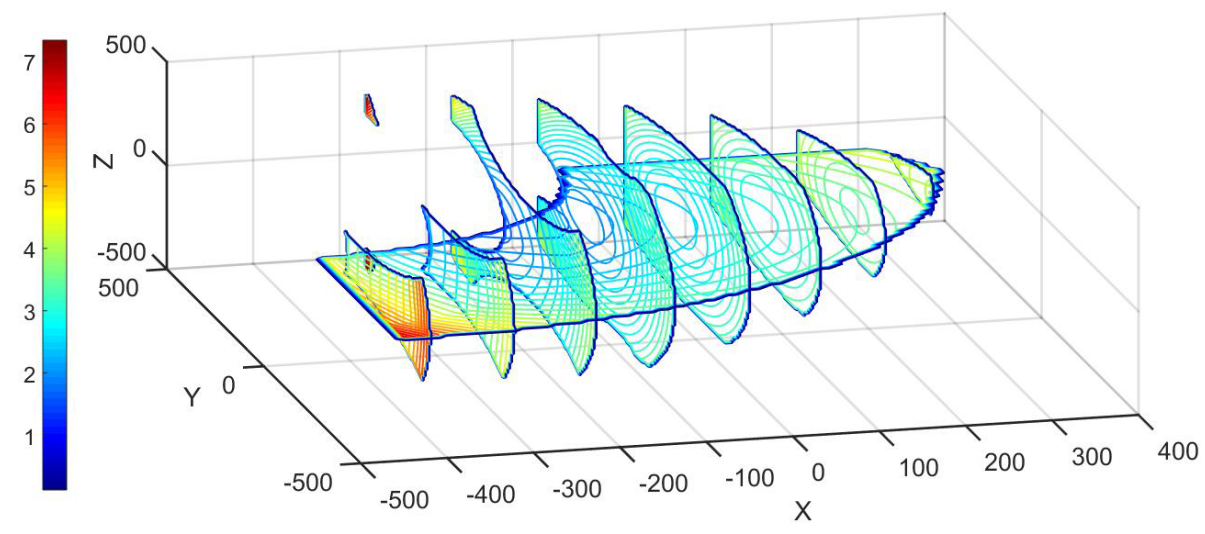

Figure 11. Condition numbers of the Jacobian matrices

screw(TWS), denoted $\$_{T_{i}}(i=1,2,3)$, is defined as a unit screw that are reciprocal to all the twist screws except the actuated one in Limb $i$. The output twist screw(OTS), denoted $\$_{O_{i}}(i=1,2,3)$, is the instantaneous movement of the moving platform when fixing all of its inputs except the one of the $i$ th limb. The input twist screw(ITS), denoted $\$_{l_{i}}(i=1,2,3)$, is the unit twist of the actuated joint in Limb $i$. And then, for the given configuration, the input transmission index (ITI) of each limb can be represented as

$\lambda_{i}=\frac{\left|\$_{T_{i}} \circ \$_{I_{i}}\right|}{\left|\$_{T_{i}} \circ \$_{I_{i}}\right|_{\max }} \quad(i=1,2,3)$

In this parallel manipulator, the translational direction of the prismatic actuator is along the limb. Thus, the input trans- 


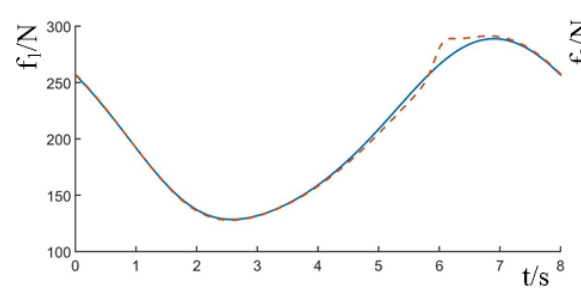

(a)

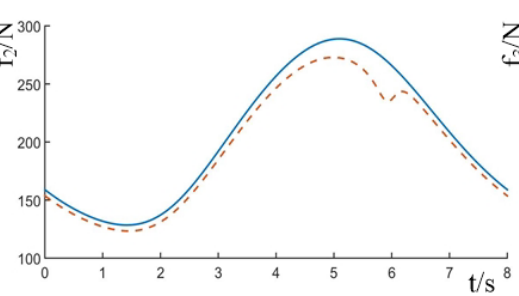

(b)

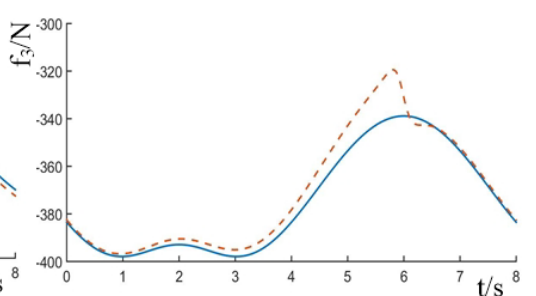

(c)

By simplified dynamic model

---- By ADAMS

Figure 12. Actuating force of each limb: (a) Actuator 1, (b) Actuator 2, (c) Actuator 3.

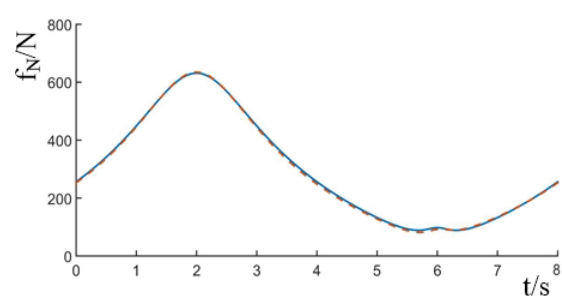

(a)

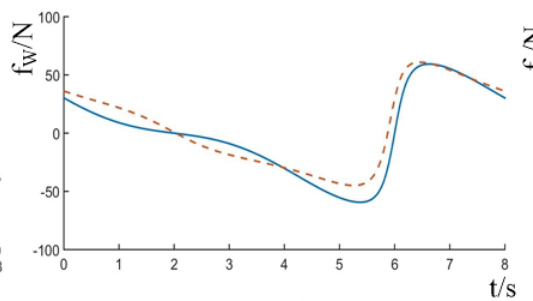

(b)

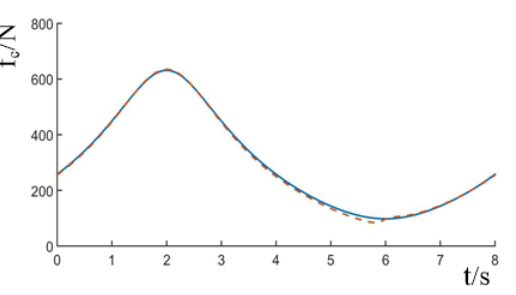

(c)

By Sect. 4.2

---- By ADAMS

Figure 13. Reaction forces of the prismatic joints: (a) $f_{N}$, (b) $f_{W}$, (c) $f_{C}$.

mission index of the limb is constant and its value is always equal to 1 . It means that this manipulator has high quality of input transmission.

Meanwhile, the output transmission index(OTI) of each limb can be represented as

$v_{i}=\frac{\left|\$_{T_{i}} \circ \$_{O_{i}}\right|}{\left|\$_{T_{i}} \circ \$_{O_{i}}\right|_{\max }}(i=1,2,3)$

The output transmission index $v_{i}$ represents the cosine of the angle between the prismatic actuator of Limb $i$ and the instantaneous movement of the moving platform. Considering ITI be always equal to 1 in this manipulator, we take the minimum value of $v_{i}$ as the local transmission index (LTI) of the whole manipulator at the given configuration, denoted as $v_{m}$. Within the whole workspace, OTIs of each limb of this manipulator can be calculated by Eq. (44). The results are presented as shown in Fig. 14a-c. And the LTIs of the whole manipulator in the workspace are as shown in Fig. 14d. It is found that LTIs in the center of the workspace are generally larger than the ones of the other areas. It illustrates that there is higher efficiency of the motion transmission when the moving platform works in this area.

The above LTIs prescribe the quality of input and output transmission in a given configuration. To further evaluate the transmissibility of the manipulator within the whole workspace, a global transmission index(GTI) of this manipulator is defined as

$\mathrm{GTI}=\frac{\int v_{\mathrm{m}} \mathrm{d} \Omega}{\int \mathrm{d} \Omega}$ where $\Omega$ is the workspace. For this manipulator, GTI over the whole workspace is 0.5664 . For the purpose of making the moving platform work in the area of better transmissibility (GTI $\geq 0.7)$, we search for the maximum area $\Gamma$ in the workspace where $\mathrm{GTI} \geq 0.7$, termed as the efficient workspace.

Find : $\Gamma \subset \Omega$

$\min :|\mathrm{GTI}-0.7|$

Through Genetic Algorithm (GA), the efficient workspace $\Gamma$ can be obtained as shown in Fig. 15. The volume of the area $\Gamma$ is $8.26 \times 10^{7} \mathrm{~mm}^{3}$, about $41.8 \%$ of the whole workspace.

\subsection{Reaction forces of the prismatic joints}

A vertical force $F_{G}=[0,-100,0]$ is exerted on the moving platform. By Eq. (23), the forces of the actuators in 3 limbs are calculated. By ratio of the results to the input force $(100 \mathrm{~N})$, the normalized reaction force of each limb is obtained. And the contour lines for them are drawn out as shown in Fig. 16a-c.

Also, we calculate the normalized reaction forces of the prismatic joints in PLMEs limb under this circumstance. Given the small distance $e$ between PLMEs limb and 2-UPR limb be $79 \mathrm{~mm}$, the length $l_{e}$ of the end link be $335 \mathrm{~mm}$, the compound reaction force $f_{\mathrm{c}}$ of the prismatic joints is figured out by Eq. (40). Similarly, by ratio of the results to $100 \mathrm{~N}$, the scale factors are obtained and presented in Fig. 16d. Obviously, when the PLMEs limb approaches the singular configuration, i.e., $\beta=\pi / 2$, the reaction forces of the prismatic 


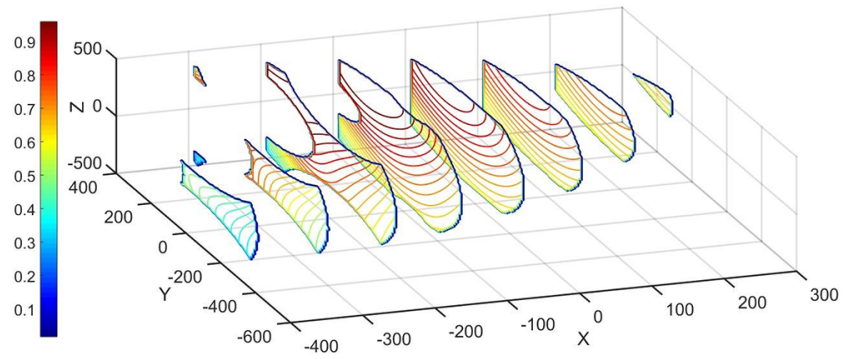

(a)

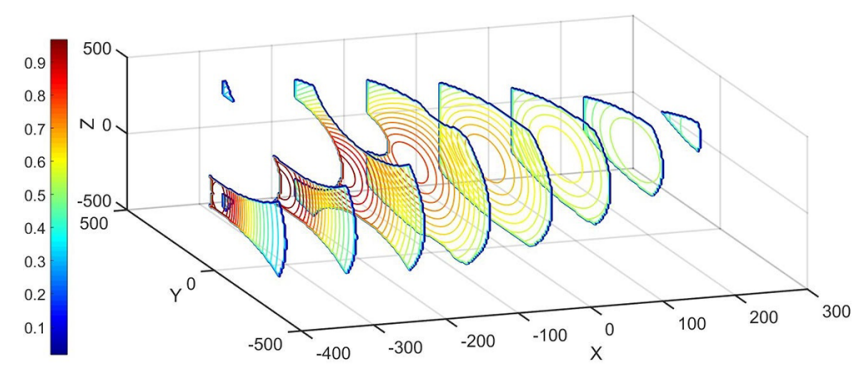

(c)

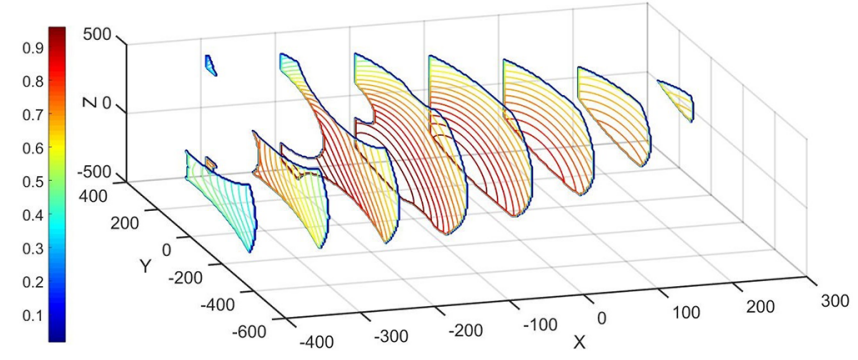

(b)

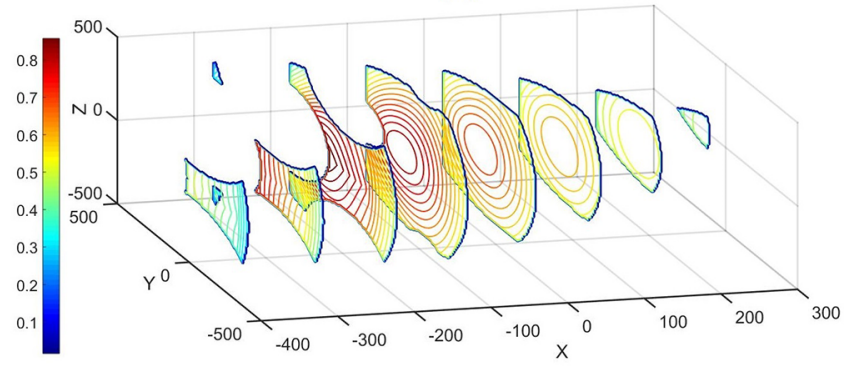

(d)

Figure 14. Local transmission index of manipulator: (a) $v_{1}$, (b) $v_{2}$, (c) $v_{3}$, (d) $v_{\mathrm{m}}$.

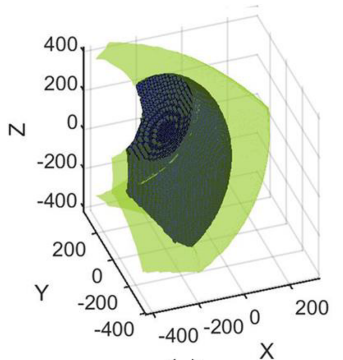

(a)

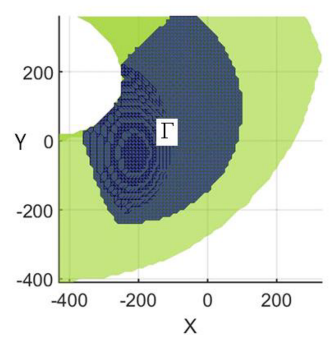

(c)

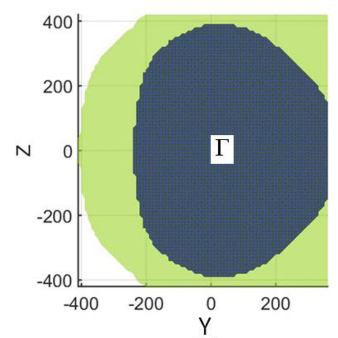

(b)

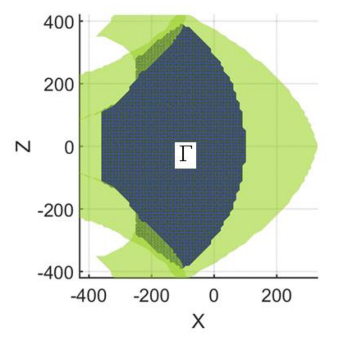

(d)
Figure 15. Efficient workspace $\Gamma$ where GTI $\geq 0.7$ : (a) oblique view, (b) front view, (c) top view, (d) side view.

joints become larger which means the force performance gets worse.

\section{Parameter optimization}

Assumed the parameters of three limbs are given, the motion range of each limb are similar with the ones mentioned above. Through changing the angles $\kappa_{1}$ and $\kappa_{2}$ as shown in Fig. 1, we can obtain different initial configurations of the whole mechanism, which are symmetrical about $x-y$ Plane. Each different initial configuration has its own workspace, transmissibility and force performance. The workspace is assumed in the range of $x>\max \left(\eta_{1}, \eta_{2}\right)$ and $y<\varsigma_{1}$ as presented in Fig. 1. To achieve the overall optimal performance, the designed parallel manipulator is expected to have large workspace, high transmission and low reaction forces.

Before optimized, we firstly draw out the workspace, transmission and normalized reaction force graphs with respect to the angles $\kappa_{1}$ and $\kappa_{2}$ within the range of $[0,1]$ and $[0, \pi / 2]$, as shown in Fig. 17a-c. From Fig. 17a, it is found that the volume of the workspace becomes large when $\kappa_{1}$ and $\kappa_{2}$ approach to zero. On the contrary, Fig. 17b and c show that the transmission and the reaction force within this area are not so good.

To make it clearly, we gathered all 3 contour maps in one graph, as show in Fig. 18, where the dashed lines represent the contour lines of the workspace volume, the solid lines represent the ones of the transmission, and the dotted lines represent the ones of the reaction force. According to the contour maps, we give the rough expected areas of workspace volume, transmission and reaction force. The overlap of the 3 areas(as shown the diagonal lines area in Fig. 18) can be regarded to be capable of optimal performance, i.e., large workspace, high transmission and low reaction force.

Based on the above analysis, a compound evaluation function $\Theta$,which involves workspace volume, transmission effi- 


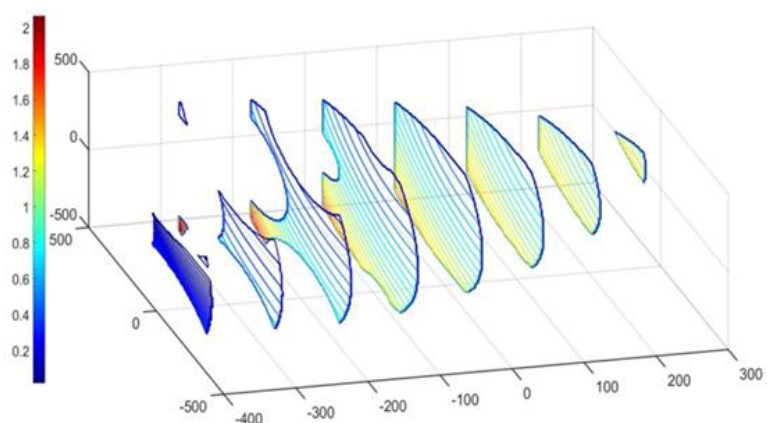

(a)

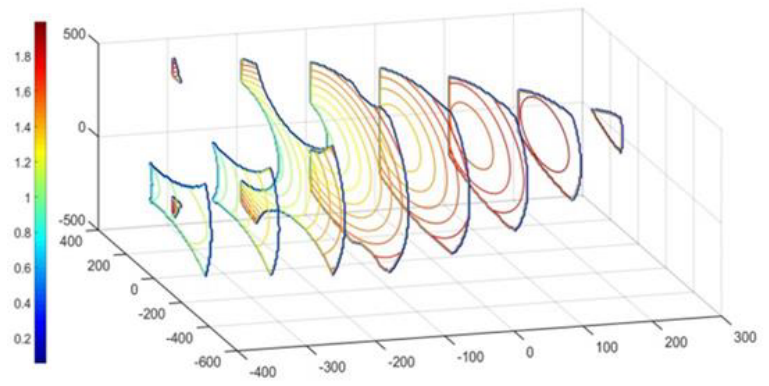

(c)

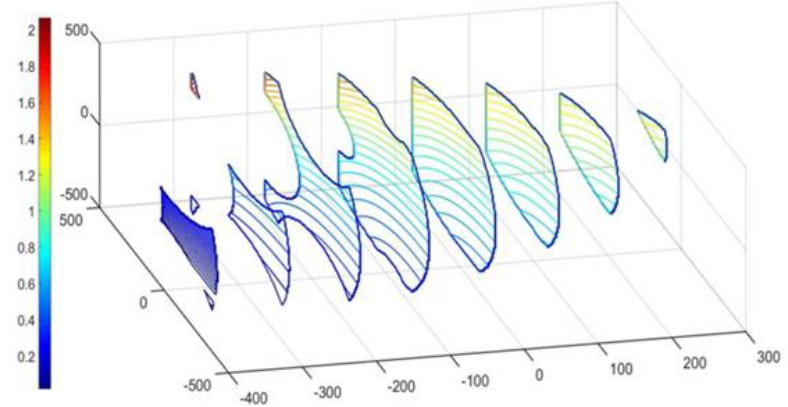

(b)

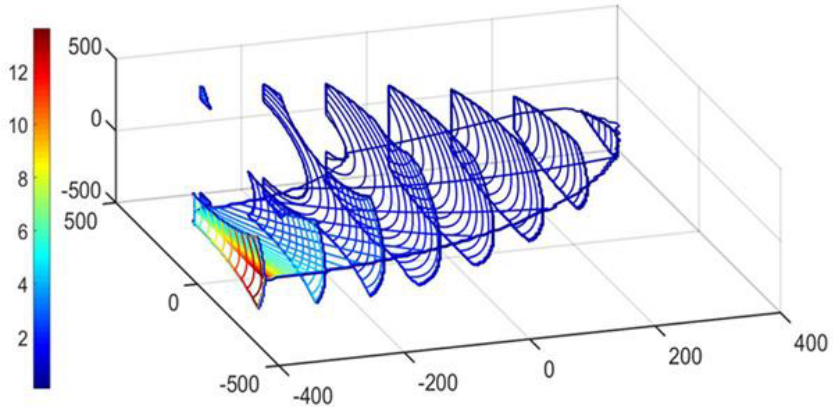

(d)

Figure 16. Scale factors of the reaction forces: (a) Limb 1, (b) Limb 2, (c) limb 3, (d) Prismatic joints.

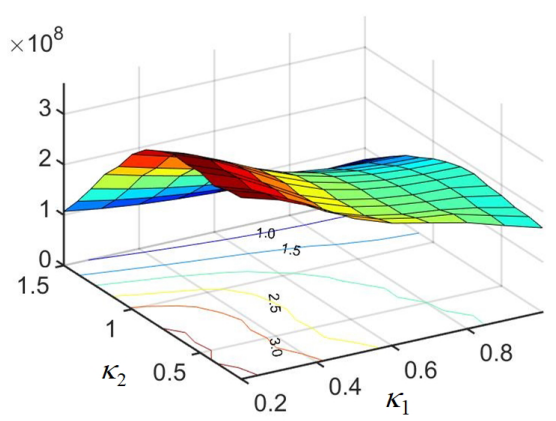

(a)

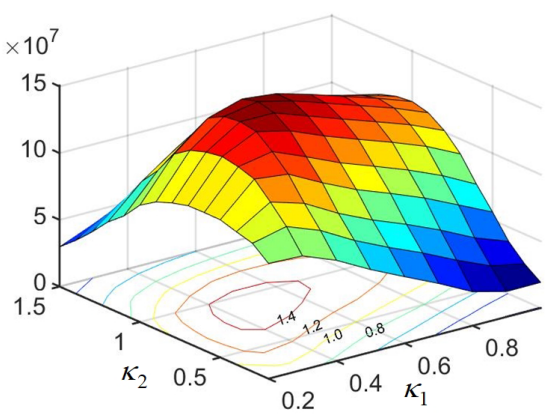

(b)

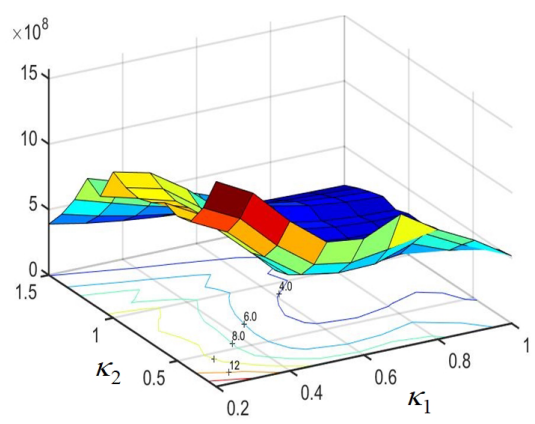

(c)

Figure 17. Performance with respect to $\kappa_{1}$ and $\kappa_{2}$ : (a) workspace volume, (b) transmission, (c) normalized reaction force.

ciency and reaction force of the manipulator, is proposed as seen in Eq. (47).

$\Theta=\frac{\left(\int v_{m} \mathrm{~d} \Omega\right)^{r} \cdot\left(\int \mathrm{d} \Omega\right)^{S}}{\left(\int f_{c} \mathrm{~d} \Omega\right)^{t}}$

where $\Omega$ is the workspace of the manipulator. $v_{m}$ is the local transmission index (LTI), which is the non-dimensional parameter. $f_{c}$ is the normalized reaction force, which is also the non-dimensional parameter. Through varying the exponents $r, s$ and $t$, we can change the weighting for certain variables to adjust the evaluation function. Usually, the exponents could be chosen as $r=1, s=1$ and $t=1$. In this case, the compound evaluation function with respect to $\kappa_{1}$ and $\kappa_{2}$ is calculated and drawn as shown in Fig. 19.

Figure 19 shows that $\Theta$ is a convex function, which obviously exists a maximum in the given range. Compared with the results of Fig. 18, it is found that the overlap almost locates in the region of the maximum of the compound evaluation function. Thus, it is feasible to obtain the optimal performance by searching the maximum of the compound evaluation function $\Theta$. Given the range of optimization variables $\kappa_{1}$ and $\kappa_{2}$ in $[0,1]$ and $[0, \pi / 2]$, the optimization is conducted by GA method. The results are obtained as $\kappa_{1}=0.605$ and $\kappa_{2}=0.781$. As shown in Figs. 18 and 19, the optimal point(red point) is located in the expected area. Compared 


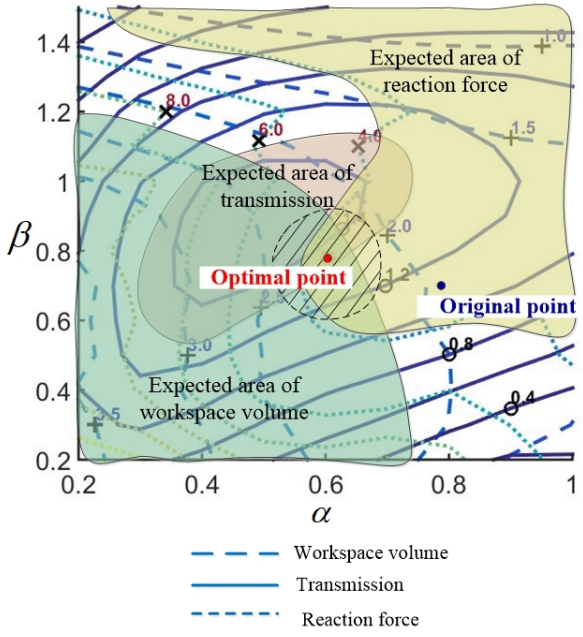

Figure 18. Contour map of overall performance.

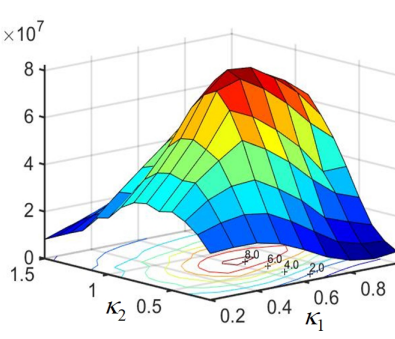

(a)

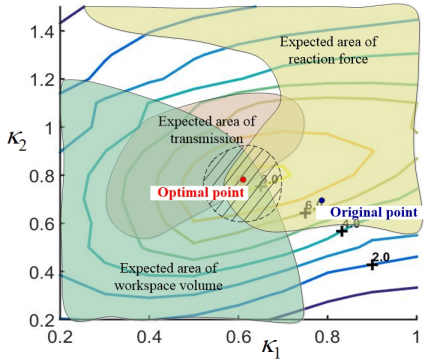

(b)
Figure 19. $\Theta$ with respect to $\kappa_{1}$ and $\kappa_{2}$ : (a) surface, (b) contour map.

with the original manipulator presented in Sect. 5 (as shown the blue point in Figs. 18 and 19), the workspace volume and transmission of the optimized manipulator are remarkably improved. Specifically, the workspace volume of the optimized manipulator is calculated as $2.1718 \times 10^{8} \mathrm{~mm}^{3}$, which is $9.9 \%$ larger than the original one. The GTI of the optimized manipulator is 0.6252 , which increase $10.3 \%$. And the volume of the efficient workspace $\Gamma$, where GTI $\geq 0.7$, is $1.2427 \times 10^{8} \mathrm{~mm}^{3}$, i.e., $60 \%$ of the whole workspace. Compared with the ones in Sect. 5, the volume of the efficient workspace of the optimized manipulator is expanded by $50 \%$, which is as shown in Fig. 20. In general, the manipulator with the optimized parameters has better overall performance than before.

\section{Prototype and Experiment}

We manufacture the prototype of this new parallel manipulator in this paper. Since the loops 2-UPR and 2-SPR are equivalent, we choose Loop 2-SPR and PLMEs limb to assemble the parallel manipulator. As shown in Fig. 21, the PLMEs limb is constructed by 2 parallel linear guides and
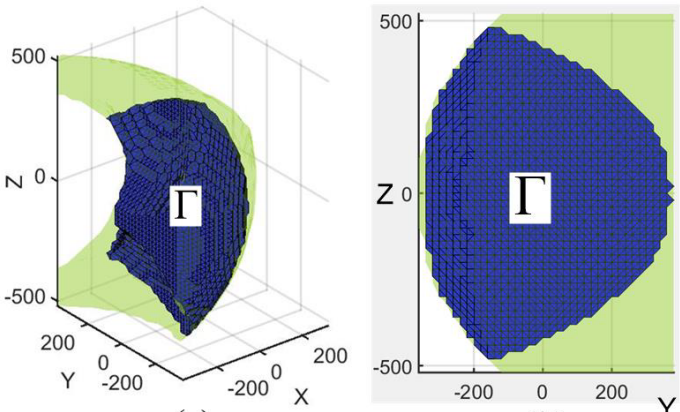

(a)

(b)

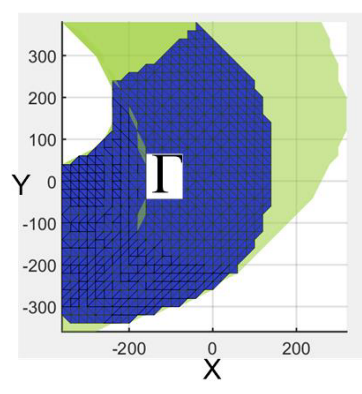

(c)

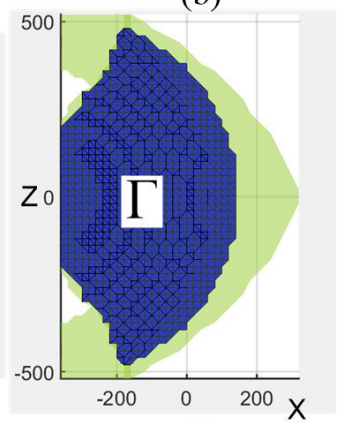

(d)
Figure 20. Efficient workspace $\Gamma$ of the optimized configuration: (a) oblique view, (b) front view, (c) top view, (d) side view.

2 sliders. The linkage $A_{1} C_{1} A_{2} C_{2}$ can slide on $B_{1}$ and $B_{2}$. And the middle link $B_{1} B_{2}$ is hinged with the fixed platform. The loop 2-SPR is constructed by 2 linear actuators. Through the " $T$ " shaped moving platform, the PLMEs limb and 2SPR limb are connected. The moving platform is driven by 3 electric linear actuators, which are controlled by the PLC(Programmable Logic Controller). Owning to the asymmetric structure, this prototype can be mounted on one side of the frame, as shown in Fig. 21.

In the experiment, we firstly control each individual linear actuator to extend and retract sequentially, as shown in Fig. 22a-c. Secondly, we make all the 3 linear actuators to extend and retract synchronously, as shown in Fig. 22d. The experiment shows that the moving platform can translate into 3 different directions. The motion of the moving platform is smoothly and continuously under the driving of the linear actuators. It proves the correctness of the mobility and motion feasibility of this kind of mechanism.

Furthermore, we plan a " $\subset$ " shaped path for the moving platform. According to the given path points, the displacement of each linear actuator relative to the initial configuration is obtained by the method proposed in Sect. 3.1. Then, input the control parameters of the linear actuators into the PLC sequentially. Under the control of PLC, the moving platform achieves the expected trajectory, as shown the red lines in Fig. 23. 


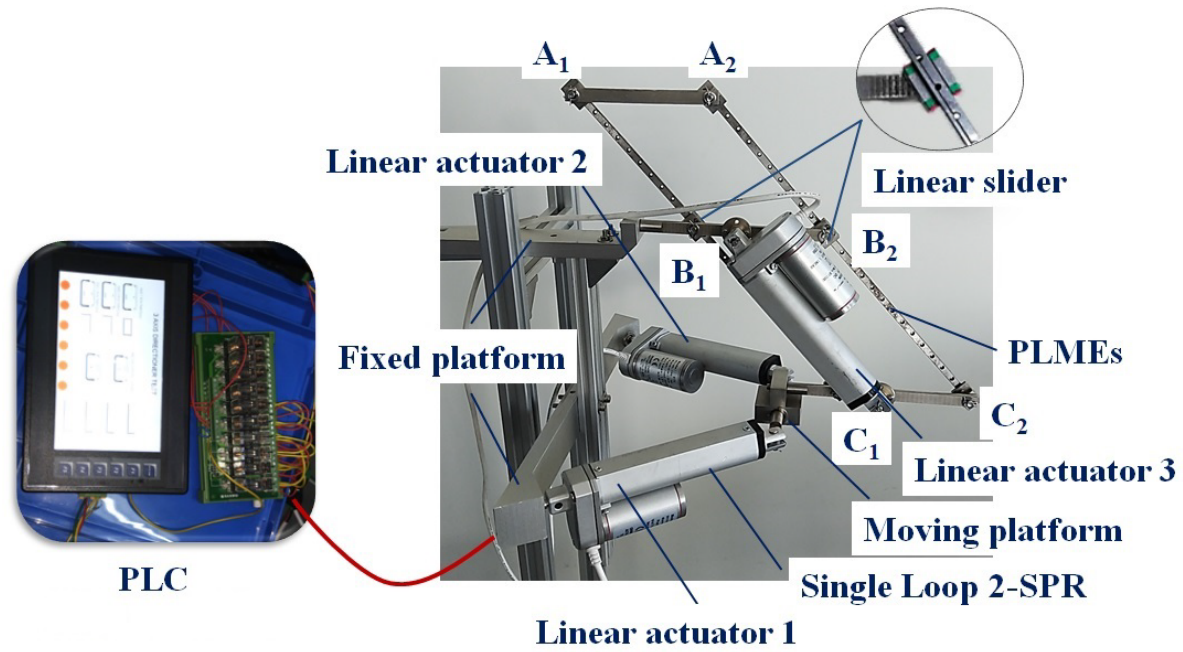

Figure 21. Prototype of new manipulator.

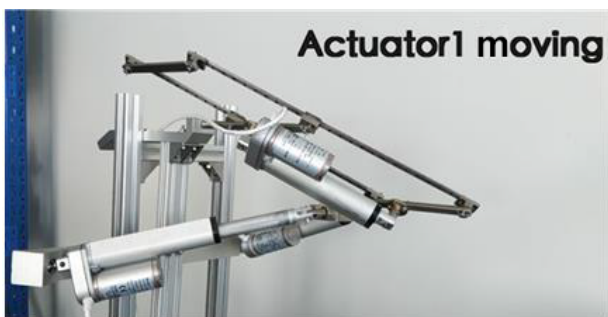

(a)



(c)

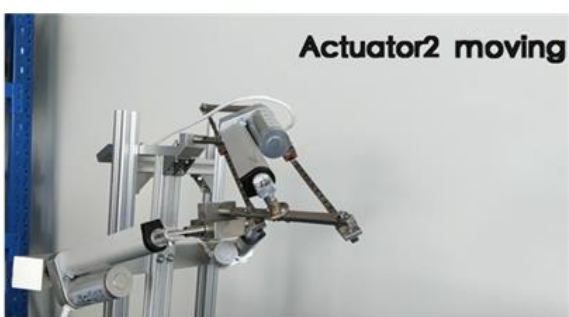

(b)

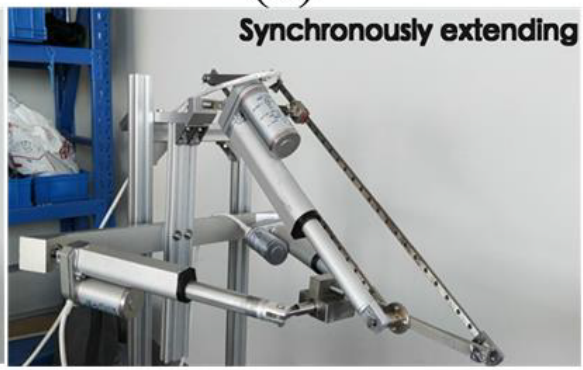

(d)

Figure 22. Motion experiments: (a) Actuator 1 moving, (b) Actuator 2 moving, (c) Actuator 3 moving, (d) Synchronously extending.

\section{Conclusions}

A new 3-DoF asymmetric translational parallel manipulator by combining of PLMEs limb and the single loop 2UPR is proposed. By the utilization of the linear actuators directly connecting the moving platform and the fixed platform, this new manipulator has higher transmission efficiency than other 3-DoF TPMs, and adapts to pick-and-place operation under heavy load. In addition, owning to asymmetric structure, this manipulator can be installed aside of the workstation, e.g. as shown in Fig. 21. It provides more flexibility in its application. In this paper, the mobility of this parallel manipulator is analyzed by screw theory and the sin- gularity is determined. Also, the simplified kinematic and dynamic models are established. And the analytic solution of the displacement and velocity equations are obtained. As the PLMEs limb is the key part of this kind of manipulator, the reaction forces of the prismatic joints in the PLMEs limb are investigated for the mechanism design. It is found that the varying of the reaction forces in the two prismatic joints are related to the Jacobian matrix of the manipulator and the configuration of the PLMEs limb, which can reveal the whole kinematic performance of the manipulator. Given an initial configuration, the workspace, condition numbers of Jacobian matrices, dynamics, motion transmission and reaction forces are discussed. Furthermore, we propose a compound evalu- 


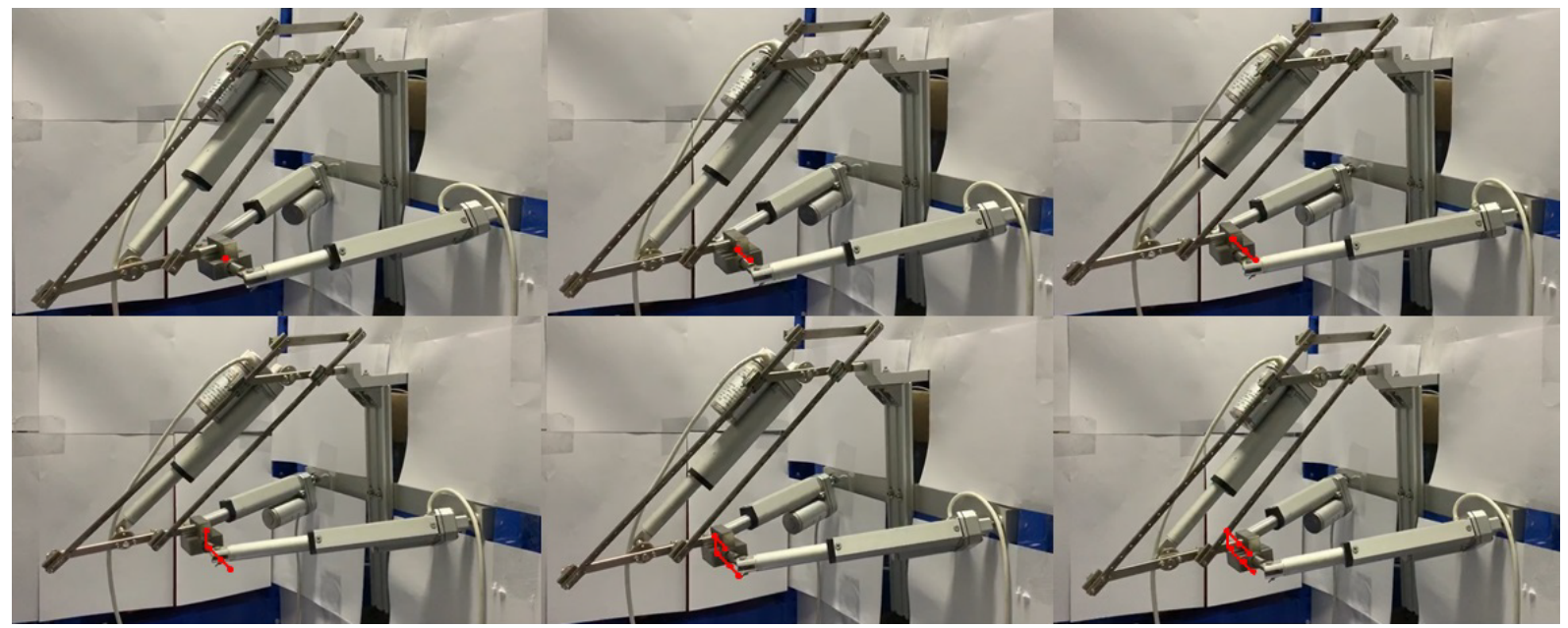

Figure 23. " $\subset$ " shaped trajectory.

ation function $\Theta$, which involves the factors of workspace volume, motion transmission and reaction force. Aiming to the maximum of $\Theta$, the optimization for the 3-DoF translational manipulator is conducted. After being optimized, the workspace volume enlarges $9.9 \%$, the GTI increases $10.3 \%$, and the volume of the efficient workspace is expanded by $50 \%$. At last, the prototype of this manipulator is manufactured, and the motion experiment validates the mobility and motion feasibility of the mechanism design.

Data availability. All the data used in this manuscript can be obtained by Yi Yang (yiyangshu@shu.edu.cn) or Yan Peng (pengyan@shu.edu.cn).

Supplement. The videos of the experiments are provided in the Supplement. The supplement related to this article is available online at: https://doi.org/10.5194/ms-10-255-2019-supplement.

Author contributions. YY conceived the overall idea of this paper and conducted the theoretical calculation as well as example studies. YT and HC performed the prototype fabrication and experiments. YP and HP verified the data and supervised the whole project. All authors discussed the results and conclusions contributing to the final manuscript.

Competing interests. The authors declare that they have no conflict of interest.

Acknowledgements. Preliminary versions of parts of this work were presented in June 2018 at 4th IEEE/IFToMM ReMAR2018 (Paper No. 33) in Delft, Netherlands, and in reference Peng et al. (2018).
Financial support. This research has been supported by the National Natural Science Foundation of China (grant nos. 51675318 and 91648119)

Review statement. This paper was edited by Guimin Chen and reviewed by two anonymous referees.

\section{References}

Blanding, D. L.: Exact Constraint: Machine Design Using Kinematic Principle, ASME Press, New York, 1999.

Bonev, I. A., Zlatanov, D., and Gosselin, C. M.: Singularity analysis of 3-dof planar parallel mechanisms via screw theory, J. Mech. Des., 125, 573-581, https://doi.org/10.1115/1.1582878, 2003.

Bouri, M. and Clavel, R.: The linear delta: Developments and applications[C]//Robotics (ISR), 2010 41st International Symposium on and 2010 6th German Conference on Robotics (ROBOTIK), VDE, 1-8, 2010.

Carricato, M. and Parenti-Castelli, V.: Singularity-free fullyisotropic translational parallel mechanisms, Int. J. Rob. Res., 21, 161-174, https://doi.org/10.1177/027836402760475360, 2002,

Chablat, D. and Wenger, P.: Architecture optimization of a 3-DOF translational parallel mechanism for machining applications, the orthoglide, IEEE. J. Robot., 19, 403-410, https://doi.org/10.1109/TRA.2003.810242, 2003.

Dai, J. S.:Geometrical Foundations and Screw Algebra for Mechanisms and Robotics, Higher Education Press, ISBN: 9787040334838, 2014.

Dai, J. S.: An historical review of the theoretical development of rigid body displacements from Rodrigues parameters to the finite twist, Mech. Mach. Theory., 41, 41-52, https://doi.org/10.1016/j.mechmachtheory.2005.04.004, 2006.

Dai, J. S., Huang, Z., and Lipkin, H.: Mobility of overconstrained parallel mechanisms, J. Mech. Des., 128, 220-229, https://doi.org/10.1115/1.1901708, 2006.

Gao, F., Yang, J. L., and Ge, Q. J.: Type synthesis of parallel mechanisms having the second class $G(F)$ sets and 
two dimensional rotations, J. Mech. Robot., 3, 011003, https://doi.org/10.1115/1.4002697, 2011.

Gogu, G.: Structural Synthesis of Parallel Robots, Part I: methodology, Springer, 2008.

Han, C., Kim, J., Kim, J., and Park, F. C.: Kinematic sensitivity analysis of the 3-UPU parallel mechanism, Mech. Mach. Theory., 37, 787-798, https://doi.org/10.1016/S0094114X(02)00021-6, 2002.

Hervé,J. M.: The Lie group of rigid body displacements, a fundamental tool for mechanism design, Mech. Mach. Theory., 34, 719—730, https://doi.org/10.1016/S0094-114X(98)00051-2, 1999.

Huang, Z. and Li, Q.: Type synthesis of symmetrical lower-mobility parallel mechanisms using constraint synthesis method, Int. J. Rob. Res., 22, 59-79, https://doi.org/10.1177/0278364903022001005, 2003.

Jin, Q. and Yang, T. L.: Theory for topology synthesis of parallel manipulators and its application to three dimensiontranslation parallel manipulators, J. Mech. Des., 126, 625-639, https://doi.org/10.1115/1.1758253, 2004.

Kong, X. and Gosselin, C. M.: Kinematics and singularity analysis of a novel type of 3-CRR 3-DOF translational parallel manipulator, Int. J. Rob. Res., 21, 791-798, https://doi.org/10.1177/02783649020210090501, 2002.

Kong, X. W. and Gosselin, C. M.: Type synthesis of 3T1R 4-DOF parallel manipulators based on screw theory, IEEE. J. Robot., 20, 181-190, https://doi.org/10.1109/TRA.2003.820853, 2004a.

Kong, X. and Gosselin,C. M.: Type synthesis of 3-DOF translational parallel manipulators based on screw theory, J. Mech. Des., 126, 83-92, https://doi.org/10.1115/1.1637662, 2004b.

Lee, J., Duffy, J., and Keler, M.: The optimum quality index for the stability of in-parallel planar platform devices, J. Mech. Des., 121, 15-22, https://doi.org/10.1115/1.2829417, 1999.

Lee, C. C. and Herve, J. M.: Type synthesis of primitive Schoenflies-motion generators, Mech. Mach. Theory, 44, 19801997, https://doi.org/10.1016/j.mechmachtheory.2009.06.001, 2009.

$\mathrm{Li}, \mathrm{Y}$. and $\mathrm{Xu}, \mathrm{Q} .:$ Stiffness analysis for a 3-PUU parallel kinematic machine, Mech. Mach. Theory, 43, 186-200, https://doi.org/10.1016/j.mechmachtheory.2007.02.002, 2008,

Li, B., Li, Y. M., Zhao, X. H., and Ge, W. M.: Kinematic analysis of a novel 3-CRU translational parallel mechanism, Mech. Sci., 6, 57-64, https://doi.org/10.5194/ms-6-57-2015, 2015.

Liu, X. J., Jay, J., and Kim, J.: A three translational DOFs parallel cube-manipulator, Robotica, 21, 645-653, https://doi.org/10.1017/S0263574703005198, 2003.

Liu, H., Huang, T., Chetwynd, D. G., and Kecskeméthy, A.: Stiffness modeling of parallel mechanisms at limb and joint/link levels, IEEE. J. Robot., 33, 734-741, https://doi.org/10.1109/TRO.2017.2654499, 2017.

Mohamed, M. G. and Duffy, J.: A direct determination of the instantaneous kinematics of fully parallel robot manipulators, J. Mech. Trans. Automation, 107, 226-229, https://doi.org/10.1115/1.3258713, 1985.
Peng, Y., Chen, H. J,, Lu, B. Z., and Yang, Y.: Design and Application of a 3-DoF Manipulator for Launch and Recovery System[C], 2018 International Conference on Reconfigurable Mechanisms and Robots (ReMAR), IEEE, 1-8, https://doi.org/10.1109/REMAR.2018.8449869, 2018.

Pierrot, F., Reynaud, C., and Fournier, A.: DELTA: a simple and efficient parallel robot, Robotica, 8, 105-109, https://doi.org/10.1017/S0263574700007669, 1990.

Tsai, L. W. and Joshi, S.: Kinematics and optimization of a spatial 3-UPU parallel manipulator, J. Mech. Des., 122, 439-446, https://doi.org/10.1115/1.1311612, 2000.

Wu, C., Liu, X. J., Wang, L., and Wang, J.: Optimal design of spherical $5 \mathrm{R}$ parallel manipulators considering the motion/force transmissibility, J. Mech. Des., 132, 031002, https://doi.org/10.1115/1.4001129, 2010.

Xie, F., Li, T., and Liu, X.: Type synthesis of 4-DOF parallel kinematic mechanisms based on Grassmann line geometry and atlas method, Chin. J. Mech. Eng-En., 26, 1073-1081, https://doi.org/10.3901/CJME.2013.06.1073, 2013.

Yang, T. L., Liu, A. X., Jin, Q., Luo, Y. F., Shen, H. P., and Hang, L. B.: Position and orientation characteristic equation for topological design of robot mechanisms, J. Mech. Des., 131, 021001, https://doi.org/10.1115/1.2965364, 2009.

Yang, Y., Peng, Y., Pu, H., and Cheng, Q.: Design of 2-Degrees-of- Freedom (Dof) Planar Translational Mechanisms With Parallel Linear Motion Elements for an Automatic Docking Device, Mech. Mach. Theory, 121, 398-424, https://doi.org/10.1016/j.mechmachtheory.2017.11.005,2018,

Yang, Y., Zhang, W., Pu, H., and Peng, Y.: A Class of Symmetrical 3T, 3T-1R, and 3R Mechanisms With Parallel Linear Motion Elements, J. Mech. Robot., 10, 051016, https://doi.org/10.1115/1.4040885, 2018.

Yang, Y., Peng, Y., Pu, H., Chen, H., Ding, X., Chirikjian, G. S., and Lyu, S.: Deployable parallel lower-mobility manipulators with scissor-like elements, Mech. Mach. Theory, 135, 226-250, https://doi.org/10.1016/j.mechmachtheory.2019.01.013, 2019.

Yu, J., Li, S., Su, H. J., and Culpepper, M. L.: Screw theory based methodology for the deterministic type synthesis of flexure mechanisms, J. Mech. Robot., 3, 031008, https://doi.org/10.1115/1.4004123, 2011.

Zhang, D., Xu, Y., Yao, J., Hu, B., and Zhao, Y. S.: Kinematics, dynamics and stiffness analysis of a novel 3DOF kinematically/actuation redundant planar parallel mechanism, Mech. Mach. Theory, 116, 203-219, https://doi.org/10.1016/j.mechmachtheory.2017.04.011, 2017.

Zhao, T. S., Dai, J. S., and Huang, Z.: Geometric Synthesis of Spatial Parallel Manipulators with Fewer Than Six Degrees of Freedom, P. I. Mech. Eng. C-JMEC, 216, 1175-1185, https://doi.org/10.1243/095440602321029418, 2002.

Zhao, Y., Wang, J., Cao, Y., Liang, B., and Zhao, T.: Constant motion/force transmission analysis and synthesis of a class of translational parallel mechanisms, Mech. Mach. Theory, 108, 57-74, https://doi.org/10.1016/j.mechmachtheory.2016.10.008, 2017. 